\title{
Generation of photonic tensor network states with circuit QED
}

\author{
Zhi-Yuan Wei $\odot,{ }^{*}$ J. Ignacio Cirac $\odot$, and Daniel Malz $\oplus^{\dagger}$ \\ Max-Planck-Institut für Quantenoptik, Hans-Kopfermann-Straße 1, D-85748 Garching, Germany \\ and Munich Center for Quantum Science and Technology (MCQST), Schellingstraße 4, D-80799 München, Germany
}

(Received 1 October 2021; revised 31 January 2022; accepted 31 January 2022; published 17 February 2022)

\begin{abstract}
We propose a circuit QED platform and protocol to generate microwave photonic tensor network states deterministically. We first show that using a microwave cavity as ancilla and a transmon qubit as emitter is a good platform to produce photonic matrix product states. The ancilla cavity combines a large controllable Hilbert space with a long coherence time, which we predict translates into a high number of entangled photons and states with a high bond dimension. Going beyond this paradigm, we then consider a natural generalization of this platform, in which several cavity-qubit pairs are coupled to form a chain. The photonic states thus produced feature a two-dimensional entanglement structure and can be interpreted as radial plaquette projected entangled pair states [Wei, Malz, and Cirac, Phys. Rev. Lett. 128, 010607 (2022)], which include many paradigmatic states, such as the broad class of isometric tensor network states, graph states, and string-net states.
\end{abstract}

DOI: 10.1103/PhysRevA.105.022611

\section{INTRODUCTION}

Producing large-scale entangled photonic states is central to many quantum technologies, including computing [1], cryptography [2], networks [3], and sensing [4]. The standard method for producing multiphoton entanglement utilizes parametric down-conversion (PDC) [5], which has been used to produce 12-photon entanglement [6]. However, PDC possesses certain limitations, most notably the exponential decrease of success probability with photon number. One promising way to overcome that is to deterministically and sequentially generate a string of entangled photons using a single quantum emitter [7-13]. The class of states that can thus be generated coincides with the set of matrix product states (MPS) [9], a type of tensor network states (TNS) that widely appears in one-dimensional quantum many-body systems [14-17]. Some sequential photon generation protocols have been experimentally realized in quantum dots [18] and circuit QED [19,20]. Using coupled emitters [21-25] or allowing the emitted photons to travel back and interact with the photon source again [26-32], it is possible to produce certain projected entangled-pair states (PEPS) [33], which are higher-dimensional generalization of MPS.

Preparing most PEPS is known to be difficult, as they generally require a preparation time that increases exponentially with the system size $[34,35]$. In contrast, a broad subset of

\footnotetext{
*zhiyuan.wei@mpq.mpg.de

†daniel.malz@mpq.mpg.de
}

Published by the American Physical Society under the terms of the Creative Commons Attribution 4.0 International license. Further distribution of this work must maintain attribution to the author(s) and the published article's title, journal citation, and DOI. Open access publication funded by the Max Planck Society.
PEPS that can be prepared efficiently are the radial plaquette PEPS (rp-PEPS) [36]. These are obtained through the sequential application of geometrically local unitaries in the form of plaquettes of side length $L_{p}$. rp-PEPS contain isometric tensor network states (isoTNS) as a subclass [37], which are PEPS [14-17] subject to an isometry condition. This immediately implies that rp-PEPS include important states such as the graph states with local connectivities [23,38], toric codes [34,39], all string-net states [40-44], and hypergraph states with local connectivities [45]. The experimental preparation of such states in two dimensions is pursued intensely [46-48].

To date, existing platforms and proposals have almost exclusively explored photonic MPS of bond dimension $D=2$ $[7,8,11,12,18,20]$, with one theoretical protocol forming an exception, which is capable of deterministically producing MPS with higher bond dimensions using an ordered array of Rydberg atoms [13]. These platforms, however, do not easily extend to produce higher-dimensional PEPS. The existing proposals that produce higher-dimensional PEPS are also mostly limited to $D=2$, and particularly focus on cluster state generation. One notable exception is the protocol in Ref. [27], which produces two-dimensional PEPS with $D>2$ utilizing the PDC process in an optical loop, but with a probabilistic protocol. Thus, despite significant efforts, there are still important theoretical challenges on deterministically producing high-fidelity and high-bond-dimension photonic tensor network states in one and particularly in higher dimensions. Sequential generation of photonic tensor network states with high bond dimension would allow creating states useful for quantum metrology [49,50], ancilla-photon superposed states [13] useful for quantum networks [51], and ground states of a large variety of many-body systems useful for quantum simulation [16,52-55].

In this work, we propose a circuit QED platform capable of deterministically generating (microwave) photonic rp-PEPS with the so-called source point [36] in one corner of the lattice. 
(a)

(b)
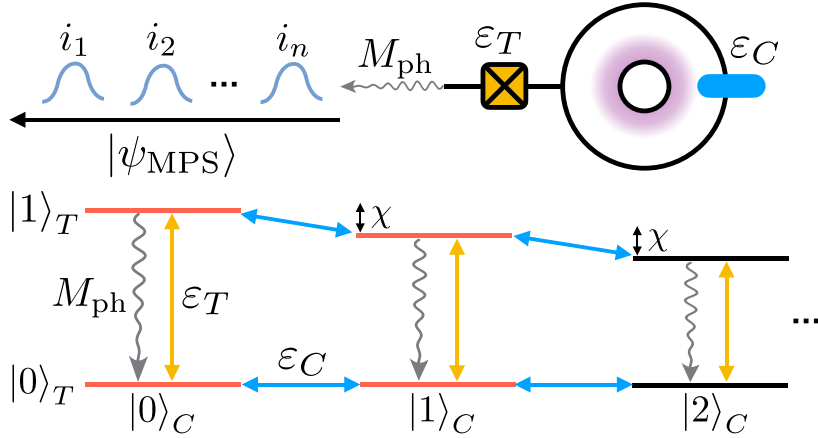

(c)

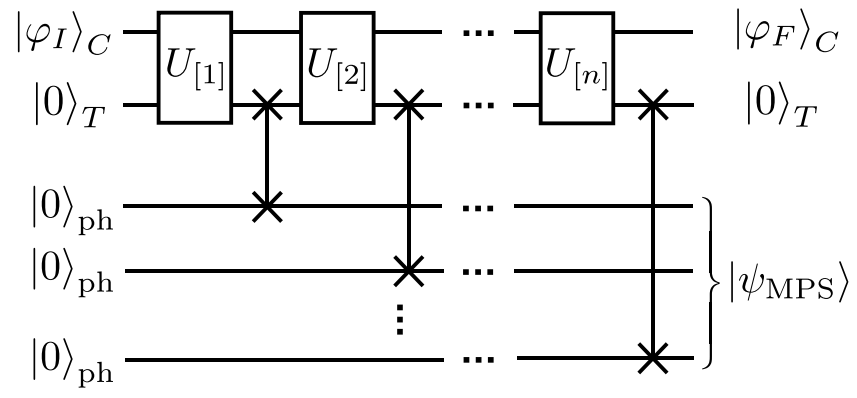

FIG. 1. Generating photonic matrix product states (MPS) with circuit QED. (a) We consider a cavity dispersively coupled to a transmon, which can be controlled by driving both the cavity $\left(\varepsilon_{C}\right)$ and the transmon $\left(\varepsilon_{T}\right)$. The transmon excitation can be converted into a traveling microwave photon. By sequentially applying unitary operations followed by photon emissions $M_{\mathrm{ph}}$, one can produce a chain of entangled photons. (b) The level diagram of the system. The red (gray) levels are used in the protocol for $D=2$ MPS generation. The yellow (light gray) arrows denote driving of the transmon, and the blue (dark gray) arrows denote driving of the cavity. (c) Quantum circuit of the MPS generation protocol. The SWAP gates correspond to photon emissions $\left(M_{\mathrm{ph}}\right)$.

We first consider a cavity dispersively coupled to a transmon qubit and show that this allows one to generate MPS of moderately high bond dimension and many entangled photons, which is outstanding among currently available platforms. Our simulations indicate that this platform has the potential to deterministically generate a one-dimensional cluster state with a large number of photons using current technologies, which would improve the experimental results in Ref. [20] severalfold. We then show that by using an array of $m$ such MPS sources, one can efficiently generate rp-PEPS. The circuit depth in terms of plaquette unitaries to prepare such a state on an $n \times m$ lattice of photons asymptotically scales as [36]

$$
\mathcal{T} \approx L_{p} n+m
$$

Since rp-PEPS is a large class, our platform allows one to create photonic states useful for applications in quantum computing [38,45], metrology [49,50,56], communication and networking [57], as well as states that exhibit topological order $[34,39,42,43]$. Moreover, the sequential nature of the protocol leads to temporally separated microwave photons, which would allow one to efficiently distribute them to multiple receivers, thus directly building a multiparty entangled state. Realization of state transfer and distribution between superconducting quantum processors is intensely pursued currently [58-60].

The rest of the article is structured as follows. In Sec. II, we present our setup to generate arbitrary MPS using a microwave cavity coupled to a transmon qubit, discuss the imperfections that arise during the MPS generation, and estimate the performance of the device. In Sec. III, we present the setup to generate photonic rp-PEPS and provide the circuits for generating the two-dimensional cluster state, the toric code, and isometric tensor network states. Finally, we analyze the scaling of the state preparation fidelity. We summarize our work in Sec. IV.

\section{GENERATING MPS WITH CIRCUIT QED}

In this section, first we introduce the setup in Sec. II A and the MPS generation protocol in Sec. II B. Then we analyze the imperfections during the protocol in Sec. II C, and show in
Sec. II D that this protocol can be implemented using current technology.

\section{A. Circuit QED sequential photon source}

We consider the setup sketched in Fig. 1(a), where a cavity (with the Hilbert space $\mathcal{H}_{C}$ ) is dispersively coupled to a transmon qubit (with the Hilbert space $\mathcal{H}_{T}$ ), with a Hilbert space $\mathcal{H}_{\text {src }}=\mathcal{H}_{T} \otimes \mathcal{H}_{C}[61]$. The transmon ground (excited) state is denoted by $|0\rangle_{T}\left(|1\rangle_{T}\right)$. Defining the transition operator $\sigma_{\alpha \beta}=|\alpha\rangle_{T}\langle\beta|$ for the transmon, the system Hamiltonian $H_{\text {src }}(t)=H_{0}+H_{\text {drive }}(t)$ contains a static part

$$
H_{0}=\omega_{T} \sigma_{11}+\omega_{C} a^{\dagger} a-\chi \sigma_{11} a^{\dagger} a,
$$

and time-dependent driving of transmon and cavity,

$$
H_{\text {drive }}(t)=\varepsilon_{C}(t) a+\varepsilon_{T}(t) \sigma_{01}+\text { H.c. }
$$

Here $\omega_{T}\left(\omega_{C}\right)$ is the frequency of the transmon qubit (cavity), and $a$ is the lowering operator of the cavity mode. The dispersive interaction strength $\chi$ sets a timescale for cavitytransmon gates. The driving amplitude of the qubit (cavity) is $\varepsilon_{T}\left(\varepsilon_{C}\right)$. The level structure of this system is shown in Fig. 1(b) [62]. This Hamiltonian gives universal control of the cavity-transmon system $[63,64]$. We assume that one can engineer the following on-demand photon emission process $M_{\mathrm{ph}}$ from the transmon excitation:

$$
M_{\mathrm{ph}}: \quad|i\rangle_{T} \rightarrow|0\rangle_{T}|i\rangle_{\mathrm{ph}}, \quad i=0,1 .
$$

For example, one can realize $M_{\mathrm{ph}}$ by coupling the qubit to an emitter via a tunable coupler [20].

\section{B. MPS generation protocol}

The setup in Sec. II A can sequentially generate photonic MPS using the generic protocol proposed in Ref. [9], schematically shown in Fig. 1(c). We identify the first $D$ Fock states of the cavity mode as our basis for the $D$-level ancilla, with a Hilbert space $\mathcal{H}_{D}$. The MPS generation protocol starts from an ancilla initial state $\left|\varphi_{I}\right\rangle_{C} \in \mathcal{H}_{D}$ with the transmon in its ground state. In each photon generation round, the ancilla first interacts with the transmon, described by a unitary operation 
$U_{[i]}$. Then, the transmon emits its excitation and returns to its ground state [denoted by a SWAP gate in Fig. 1(c)], generating a photonic qubit defined by the presence or absence of a photon at that time.

Compared to the setup in Ref. [10] which proposes to employ a $D$-level atom as ancilla and an optical cavity as emitter, our setup is better suited to current circuit QED experiments. In particular, our protocol exploits the long coherence times that can be achieved in microwave cavities and uses transmon qubits only to control and emit, but not to store excitations.

Throughout the protocol, unitaries are applied only after emission of the photon from the emitter, such that they always act on ancilla-transmon states of form $|\varphi\rangle_{C}|0\rangle_{T}$. As a result we can specify the action of the unitaries in terms of isometries $V$ that act only on the cavity Hilbert space:

$$
U_{[i]}\left(|\varphi\rangle_{C}|0\rangle_{T}\right)=\sum_{j=0}^{1}\left(V_{[i]}^{j}|\varphi\rangle_{C}\right)|j\rangle_{T} .
$$

Since $U_{[i]}$ is unitary, the matrices $\left\{V_{[i]}^{j}\right\}$ satisfy the isometry condition $\sum_{j=0}^{1} V_{[i]}^{j \dagger} V_{[i]}^{j}=I_{D}$. The quantum state after $n$ rounds of photon generation is $|\Psi\rangle=$ $M_{\mathrm{ph}} U_{[n]} \cdots M_{\mathrm{ph}} U_{[1]}\left|\varphi_{I}\right\rangle_{C}$. By disentangling the ancilla and the photonic state in the last step, we obtain the final state $|\Psi\rangle=\left|\varphi_{F}\right\rangle_{C} \otimes\left|\psi_{\text {MPS }}\right\rangle$, which includes the following photonic MPS [9]:

$$
\left|\psi_{\mathrm{MPS}}\right\rangle \propto \sum_{i_{1}, \ldots, i_{n}=0}^{1} C\left\langle\varphi_{F}\left|V_{[n]}^{i_{n}} \cdots V_{[1]}^{i_{1}}\right| \varphi_{I}\right\rangle_{C}\left|i_{n} \cdots i_{1}\right\rangle .
$$

We use the quantum optimal control (QOC) $[13,65]$ to find the pulse sequences that implement the desired unitary operations for our protocol (see details in Appendix A). As a demonstration, we show how to generate a linear cluster state [66], which can be written as an MPS of bond dimension $D=2$, where

$$
\begin{aligned}
V_{[i]}^{0} & =\frac{1}{\sqrt{2}}\left(\begin{array}{ll}
1 & 0 \\
1 & 0
\end{array}\right), \quad V_{[i]}^{1}=\frac{1}{\sqrt{2}}\left(\begin{array}{cc}
0 & 1 \\
0 & -1
\end{array}\right), \\
\left|\varphi_{I}\right\rangle_{C} & =\frac{1}{\sqrt{2}}(|0\rangle+|1\rangle), \quad\left|\varphi_{F}\right\rangle_{C}=|0\rangle .
\end{aligned}
$$

In each round except the last, we apply the same unitary $U_{[i \neq n]}$ followed by photon emission represented by $M_{\mathrm{ph}}$, which adds one site to the state. In the last step, we apply the unitary $U_{[n]}$ followed by $M_{\mathrm{ph}}$, which emits the last photon and disentangles the source from the photons. The pulse sequence of the driving [Eq. (3)] for implementing the two unitaries is shown in Fig. 2, and we provide more details in Appendix A.

\section{Analysis of experimental imperfections}

Ideally, the above protocol would generate the desired pure photonic state $\left|\psi_{\text {MPS }}\right\rangle$. However, various errors may occur in this system during the unitary operation and the photon emission process. Thus the protocol produces an $n$ photon density matrix $\rho_{\text {ph }}$, with a nonunit fidelity $\mathcal{F}_{\text {MPS }}=$ ph $\left\langle\psi_{\text {MPS }}\left|\rho_{\text {ph }}\right| \psi_{\text {MPS }}\right\rangle_{\text {ph }}$. Due to the sequential nature of the protocol, $\mathcal{F}_{\text {MPS }}$ is an exponentially decaying function of the
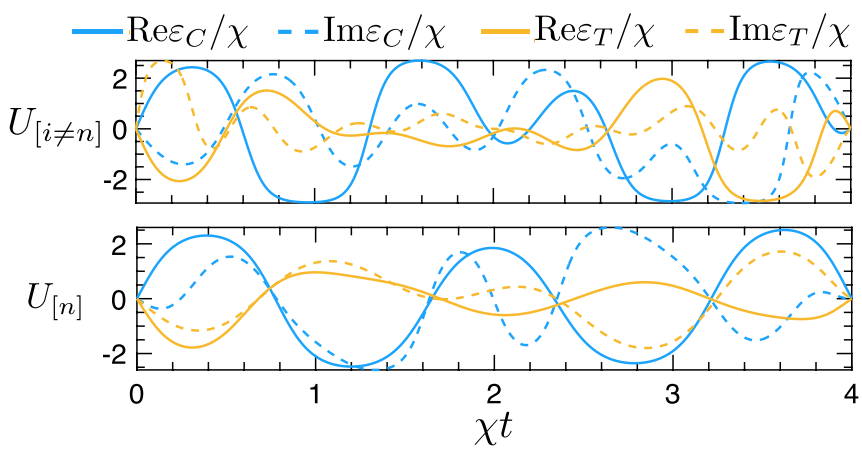

FIG. 2. Optimized cavity and transmon drive [Eq. (3)] to implement the unitaries $U_{[i \neq n]}$ and $U_{[n]}$ for the cluster state [Eq. (7)] generation.

emitted photon number $n$, that, is

$$
\mathcal{F}_{\text {MPS }}=e^{-\xi n},
$$

where $\xi$ is the error per photon emission. An example of this behavior is shown in Fig. 3(a).

Decoherence processes in the cavity-transmon system include transmon decay at a rate $\Gamma_{T}$, cavity mode decay at a rate $\Gamma_{C}$, and transmon dephasing at a rate $\Gamma_{\phi}[67,68]$. These processes happen during both the unitary operations and the photon emission process. The finite anharmonicity $\alpha$ of the transmon further allows leakage into the second excited state $|2\rangle_{T}$ in every unitary operation.

To model the imperfections due to finite anharmonicity $\alpha$ during unitary operations, we model the transmon as a truncated anharmonic oscillator with basis $\left\{|0\rangle_{T},|1\rangle_{T},|2\rangle_{T}\right\}$. After further including the decoherence effects, the system density matrix $\rho_{\text {src }} \in \mathcal{H}_{\text {src }}$ evolves under the master equation

$$
\begin{aligned}
\dot{\rho}_{\mathrm{src}}(t)= & -i\left[H_{\mathrm{src}}^{\prime}(t), \rho_{\mathrm{src}}(t)\right] \\
& +\sum_{n=T, C, \phi}\left(J_{n} \rho_{\mathrm{src}}(t) J_{n}^{\dagger}-\frac{1}{2}\left\{\rho_{\mathrm{src}}(t), J_{n}^{\dagger} J_{n}\right\}\right),
\end{aligned}
$$
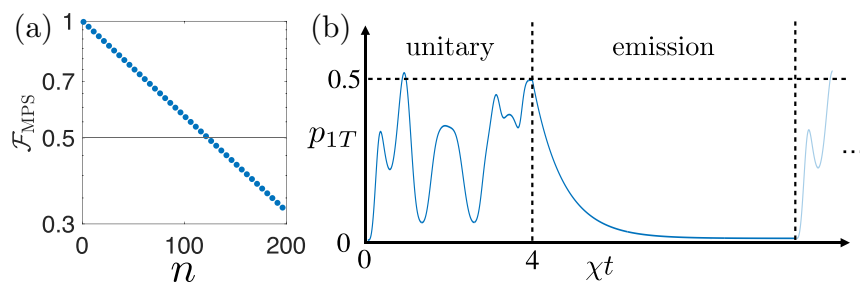

FIG. 3. (a) The state fidelity versus the number of photons for the cluster state generated using the pulse sequence in Fig. 2, with the state-of-the-art parameters listed in Sec. II D. The horizontal line denotes $\mathcal{F}_{\text {MPS }}=1 / 2$, with the corresponding photon number $N_{\text {ph }}$ defined as the entanglement length of the photon string. (b) Evolution of the transmon excited population $p_{1 T}$ during one sequence of cluster state generation. The $p_{1 T}$ shows a transient evolution during the unitary operation $U_{[i \neq n]}$ driven by the pulse in Fig. 2, and exponentially decays during the photon emission process. 
with $H_{\mathrm{src}}^{\prime}(t)$ being the system Hamiltonian including transmon double excitations with anharmonicity $\alpha$. Defining the spin operators $\sigma_{i j}=|i\rangle_{T}\langle j|$, the jump operators are $J_{T}=\sqrt{\Gamma_{T}}\left(\sigma_{01}+\sqrt{2} \sigma_{12}\right), J_{C}=\sqrt{\Gamma_{C}} a$, and $J_{\phi}=$ $\sqrt{\Gamma_{\phi}}\left(\sigma_{11}+2 \sigma_{22}\right)$.

Since current experiments generally have $|\alpha| \gg|\chi| \gg$ $\Gamma_{C}, \Gamma_{T}, \Gamma_{\phi}[61,67,69]$, for a gate time $T \sim 1 /|\chi|$ we can estimate the scale of the errors on $\mathcal{F}_{\text {MPS }}$ during each unitary operation perturbatively. The total error $\xi$ is a sum of several parts: (i) transmon decay $\xi_{\Gamma_{T}}$, (ii) transmon dephasing $\xi_{\Gamma_{\phi}}$, (iii) cavity decay $\xi_{\Gamma_{C}}$, and (iv) transmon nonlinearity $\xi_{\alpha}$. These contributions scale as

$$
\begin{array}{ll}
\xi_{\Gamma_{T}} \sim \Gamma_{T} /|\chi|, & \xi_{\Gamma_{\phi}} \sim \Gamma_{\phi} /|\chi|, \\
\xi_{\Gamma_{C}} \sim \Gamma_{C} /|\chi|, & \xi_{\alpha} \sim|\chi|^{2} /|\alpha|^{2} .
\end{array}
$$

Imperfections also affect the photon emission process. First, there is a finite photon retrieval efficiency $p_{\mathrm{em}}$ of the emitter [70] associated to $M_{\mathrm{ph}}$. Second, system decoherence happens during the photon emission. We assume a finite photon emission rate $\Gamma_{\mathrm{em}}$, and thus a finite duration of photon emission, $T_{\mathrm{em}}$ (which we can tune). In the regime of $\Gamma_{T}, \Gamma_{\phi}, \Gamma_{C} \ll \Gamma_{\text {em }}$, we can estimate scaling of error on $\mathcal{F}_{\text {MPS }}$ due to system decoherences during each emission process as

$$
\begin{aligned}
& \xi_{\Gamma_{C}}^{\mathrm{em}} \sim \Gamma_{C} T_{\mathrm{em}}, \quad \xi_{\Gamma_{T}}^{\mathrm{em}} \sim \Gamma_{T} / \Gamma_{\mathrm{em}}, \\
& \xi_{\Gamma_{\phi}}^{\mathrm{em}} \sim \Gamma_{\phi} / \Gamma_{\mathrm{em}}, \quad \xi_{p_{\mathrm{em}}} \sim-\ln p_{\mathrm{em}} .
\end{aligned}
$$

Notably, here $\xi_{\Gamma_{T}}^{\mathrm{em}}$ and $\xi_{\Gamma_{\phi}}^{\mathrm{em}}$, which are due to transmon decoherence, do not depend on the emission time $T_{\mathrm{em}}$. The expression for $\xi_{p_{\mathrm{em}}}$ comes from the fact that $e^{-\xi_{\mathrm{pem}} n_{\mathrm{ph}}} \sim p_{\mathrm{em}}^{n_{\mathrm{ph}}}$. The finite photon emission time also results in a residual population of the transmon first excited state $p_{1 T}\left(T_{\mathrm{em}}\right)=$ $e^{-\Gamma_{\mathrm{em}} T_{\mathrm{em}}} p_{1 T}(0)$, which reduces the photon retrieval efficiency $p_{\text {em }}$. The whole photon emission process including all imperfections can be described by a process map $W_{\mathrm{ph}}: \mathcal{H}_{\mathrm{src}} \rightarrow$ $\mathcal{H}_{\text {src }} \otimes \mathcal{H}_{\text {ph }}$ that maps $\rho_{\text {src }}$ to a system-photon joint density matrix (see the construction of $W_{\mathrm{ph}}$ in Appendix C). Finally, note that we did not include the imperfections during photon transmission, which is not a part of our model.

Using the solution of Eq. (9) and the process map $W_{\mathrm{ph}}$, we can use a matrix product density operator (MPDO) approach [13] to obtain the photonic state fidelity $\mathcal{F}_{\text {MPS }}$ and extract the overall error rate $\xi$ (details in Appendix B). As a demonstration, we analyze the process of generating the one-dimensional cluster state using the pulses in Fig. 2. From the scaling data of $\xi$ as a function of various imperfections (details in Appendix D), in the regime of small error $(\xi \ll 1)$, we have

$$
\xi \approx \xi_{\text {unit }}+\xi_{\mathrm{em}}^{\mathrm{src}}+\xi_{\mathrm{em}}^{\mathrm{ph}},
$$

which includes imperfections during unitary operation,

$$
\xi_{\text {unit }}=\beta_{0}+\frac{\beta_{C} \Gamma_{C}+\beta_{T} \Gamma_{T}+\beta_{\phi} \Gamma_{\phi}}{|\chi|}+\frac{\beta_{\alpha}|\chi|^{2}}{|\alpha|^{2}},
$$

cavity-transmon decoherence during photon emission,

$$
\xi_{\mathrm{em}}^{\mathrm{src}}=\beta_{\phi, \mathrm{em}} \Gamma_{\phi} / \Gamma_{\mathrm{em}}+\beta_{C, \mathrm{em}} \Gamma_{C} T_{\mathrm{em}},
$$

and imperfections of the photon emission,

$$
\xi_{\mathrm{em}}^{\mathrm{ph}}=-\beta_{\mathrm{em}} \ln \left(\left(1-e^{-\Gamma_{\mathrm{em}} T_{\mathrm{em}}}\right) \frac{\Gamma_{\mathrm{em}}}{\Gamma_{\mathrm{em}}+\Gamma_{T}} p_{\mathrm{em}}\right) .
$$

The above overall scaling matches our qualitative prediction Eqs. (10) and (11) very well. The nonuniversal coefficients $\left\{\beta_{i}\right\}$ depend on the target photonic state and the pulse shape, and are extracted from scaling data shown in Appendix C. In our example of cluster state generation, using the pulses shown in Fig. 2, we obtain

$$
\begin{aligned}
& \beta_{C}=2.20, \quad \beta_{T}=1.43, \quad \beta_{\phi}=0.92, \\
& \beta_{\phi, \mathrm{em}}=0.51, \quad \beta_{\mathrm{C}, \mathrm{em}}=0.47, \quad \beta_{\mathrm{em}}=0.47, \\
& \beta_{0}=2.58 \times 10^{-4}, \quad \beta_{\alpha}=46.7 .
\end{aligned}
$$

Here $\beta_{0}$ corresponds to the imperfect synthesis of the optimal control pulse, and can typically be made negligible. All other $\left\{\beta_{i}\right\}$ are of order $O(1)$, except $\beta_{\alpha}$ which correspond to the effect of transmon double excitations, which is particularly large because it scales with the maximum driving amplitude of the transmon, which can be several times larger than $|\chi|$ (cf. Fig. 2). Note that the decoherence due to transmon double excitations [cf. Eq. (13)] is still relatively small, thanks to the strong anharmonicity $|\alpha|$ that suppresses the transmon double excitations, and one can further reduce this leakage error by including higher levels in the pulse optimization [71].

\section{Performance estimation of protocol}

We estimate the performance of this sequential photon source using current state-of-the-art experimental parameters. Specifically, we use cavity and transmon parameters [67] $\Gamma_{C}=0.37 \mathrm{kHz}, \Gamma_{T}=5.88 \mathrm{kHz}, \Gamma_{\phi}=23.26 \mathrm{kHz}, \alpha=2 \pi \times$ $-236 \mathrm{MHz}$, and $\chi=2 \pi \times-2.194 \mathrm{MHz}$, and the photon emission parameters [20] $\Gamma_{\mathrm{em}}=2 \pi \times 1.95 \mathrm{MHz}$ and $p_{\mathrm{em}}=$ 1 [72]. We choose the photon emission duration as $T_{\mathrm{em}}^{\mathrm{opt}}=$ $\ln \left(1+\beta_{\mathrm{em}} \Gamma_{\mathrm{em}} / \beta_{\mathrm{C}, \mathrm{em}} \Gamma_{C}\right) / \Gamma_{\mathrm{em}}$ to minimize $\xi$ [cf. Eq. (12)] under the assumption of a fixed photon emission rate $\Gamma_{\mathrm{em}}$. For the one-dimensional cluster state generated using the pulse sequence in Fig. 2, we plot the resulting fidelity $\mathcal{F}_{\text {MPS }}$ in Fig. 3(a). Defining the entanglement length $N_{\text {ph }}=\ln 2 / \xi$ [for which $\xi$ has been defined in Eq. (8)], which is the photon number at which the fidelity drops down to $\mathcal{F}_{\text {MPS }}=1 / 2$, we obtain $N_{\text {ph }} \approx 123$, which would mean an eightfold increase compared to the experimentally demonstrated $N_{\mathrm{ph}} \approx 15$ in Ref. [20]. This improvement partly comes from the fact that we exploit the long transmon lifetime reported in Ref. [67]. If we use the transmon properties reported in Ref. [20], our protocol gives $N_{\mathrm{ph}} \approx 47$ (see Appendix D), which is still a substantial improvement.

To understand this improvement, we plot the population $p_{1 T}$ of the transmon excited state $|1\rangle_{T}$ during one photon generation round in Fig. 3(b). We see that $|1\rangle_{T}$ is only transiently populated during the unitary operation $U_{[i \neq n]}$ (driven by the pulse in Fig. 2), compared to the protocol in Ref. [20] where one always has $p_{1 T}=1 / 2$ during the cluster state generation. This leads to a substantial improvement of the entanglement length obtained in our protocol [73]. Moreover, it is possible to further reduce $p_{1 T}$ by adding a corresponding penalty in the optimal control algorithm (cf. Appendix A). 
Finally, we estimate the scaling of $N_{\mathrm{ph}}$ with the bond dimension $D$ of the desired MPS as (see Appendix D 3)

$$
N_{\text {ph }} \propto D^{-2},
$$

and this scaling mainly comes from the time $T_{\mathrm{MPS}}^{D}=O\left(D^{2}\right)$ [74] taken to implement a unitary on the $2 D$-dimensional Hilbert space $\operatorname{dim} \mathcal{H}_{\text {src }}=2 D$ (cf. Sec. II B). This scaling indicates that our system can create MPS of moderate bond dimensions. This already finds many applications and can efficiently capture the ground states of one-dimensional local gapped Hamiltonians [52-55].

\section{GENERATING RP-PEPS WITH CIRCUIT QED}

The previous section shows that the proposed cavitytransmon system can produce high-fidelity one-dimensional photonic MPS. In this section, we demonstrate how to extend this to implement the high-dimensional photonic state generation protocol introduced in Ref. [36]. First, in Sec. III A we introduce the array of coupled sequential photon sources and prove the universality of the Hamiltonian for this system, which allows this system to implement arbitrary local unitary transformations. Then we show in Sec. III B that, using this setup, one can generate rp-PEPS, whose source point (cf. Sec. III B) sits on a corner of the lattice. After that, in Sec. III C we demonstrate this protocol by discussing the preparation of two-dimensional cluster states, the toric code, and the isoTNS. Finally, we analyze the scaling of the state preparation fidelity for this protocol in Sec. III D. Here we mainly focus on generating two-dimensional photonic states; however, this protocol readily extends to higher spatial dimensions [36].

\section{A. Setup: Array of sequential photon sources}

Let us consider a natural generalization of the setup in Sec. II A to a quasi-one-dimensional array of $L_{c} \times m$ cavitytransmon pairs, and use the first $D^{\prime}$ Fock states of each cavity. The $L_{c}$ cavities in each row form an ancilla $A$ of dimension $D=D^{\prime L_{c}}$. While $L_{c}=1$ is the most straightforward choice, sometimes it may be beneficial to choose $L_{c}>1$, as we comment on in Sec. IIID. Moreover, in each row there is one emitter $\left\{T_{j}\right\}_{j=1, \ldots, m}$ [see Fig. 4(a)], while other transmon qubits are used to provide universal control to the corresponding cavities [75].

We couple each neighboring pair of photon sources by a coupler that interacts with both cavities [76] [shown as green boxes in Fig. 4(a)]. By driving the coupler with two-tone pumps, the four-wave mixing process of the coupler reduces to the following bilinear interaction [77]:

$$
H_{\mathrm{int}}^{i j}(t)=g_{i j}(t)\left(e^{i \varphi_{i j}(t)} a_{i}^{\dagger} a_{j}+\text { H.c. }\right) \text {. }
$$

Here $a_{i}^{\dagger}$ denotes the creation operator of the cavity for the $i$-th photon source. The coupling strength $g_{i j}(t)$ and phase $\varphi_{i j}(t)$ can be controlled through drives. Let us denote the set of vertices of the array of sources in Fig. 4(a) as $\mathcal{V}$, where each vertex $v_{i j}$ connects the cavities of the $i$ th and $j$ th cavitytransmon pairs. One can thus write down the Hamiltonian of

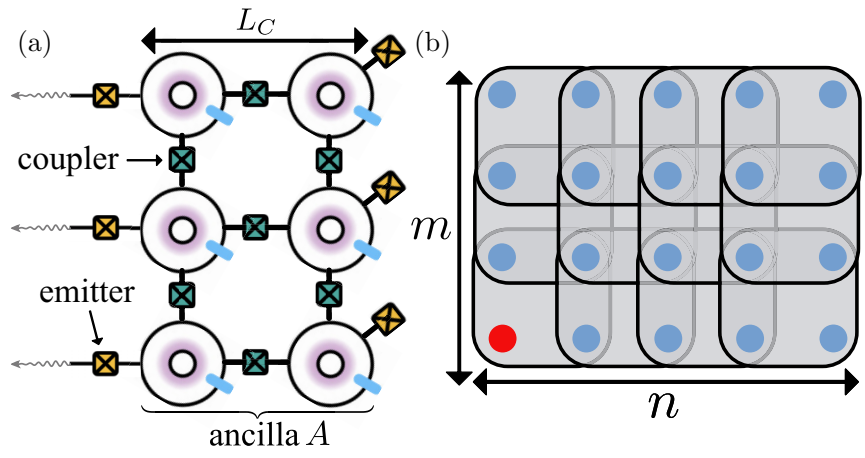

FIG. 4. (a) The setup to generate photonic rp-PEPS, which consist of a quasi-one-dimensional array of cavity-transmon pairs [the notations follow that in Fig. 1(a)]. In each row there is a transmon emitter that can emit photons. The neighboring cavities are connected by Y-shaped couplers [the green (dark gray) boxes]. (b) rp-PEPS with open boundary conditions are produced by sequentially applying unitaries on overlapping regions. Here the source point [36] of the state is denoted by the red dot.

this system as

$$
H_{\text {array }}(t)=\sum_{i=1}^{L_{c} \times m} H_{\mathrm{src}}^{i}(t)+\sum_{v_{i j} \in \mathcal{V}} H_{\mathrm{int}}^{i j}(t),
$$

where $H_{\mathrm{src}}^{i}$ is the Hamiltonian for the $i$-th source, containing the terms in Eqs. (2) and (3). Equipped with the universal control of each source and the bilinear couplings, we prove in Appendix $\mathrm{F}$ that $H_{\text {array }}$ provides universal control. Given this, we can assume that one can implement arbitrary local unitary operations on this system.

In sum, this system can be represented by a onedimensional array of $D^{\prime L_{C}}$-level ancillas (labeled by $\left\{A_{j}\right\}_{j=1, \ldots, m}$ ) coupled to transmon emitters (labeled by $\left.\left\{T_{j}\right\}_{j=1, \ldots, m}\right)$, illustrated in Fig. 5. Note that one can let more transmons in each row emit photons, which effectively increases the dimension of the transmon emitters. Finally, in the next sections we will treat each ancilla effectively as $L_{p}-1$ qubits by choosing $D^{\prime}$ and $L_{c}$ such that

$$
D^{\prime L_{C}} \geqslant 2^{L_{p}-1} \text {. }
$$

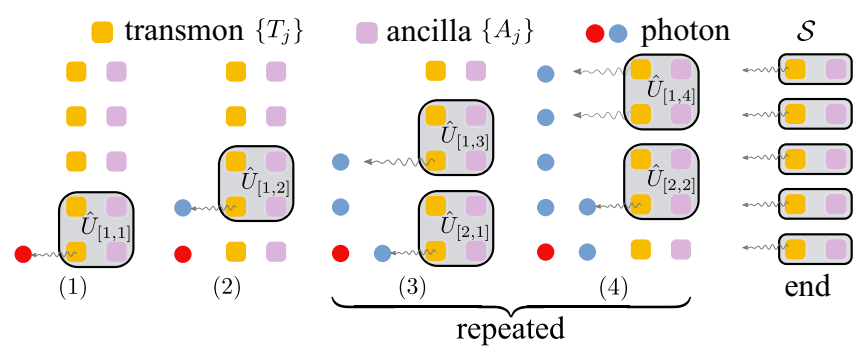

FIG. 5. The preparation of photonic rp-PEPS $\left(L_{p}=2\right.$ here). In the preparation of the $(i, j)$ th site, we apply a unitary $\hat{U}_{[i, j]}$ followed by a photon emission of the transmon $T_{j}$. After the initial steps (1) and (2), steps (3) and (4) will be repeated. At the end of the protocol, we swap the excitations on the ancillas to the emitters and then convert them to photons, denoted as $\mathcal{S}$. 


\section{B. Preparation of rp-PEPS}

Reference [36] introduces a generic protocol to produce rp-PEPS on flying qubits. rp-PEPS are states prepared by sequentially applying unitaries on plaquettes of size $L_{p} \times L_{p}$ $\left(L_{p} \ll n, m\right)$ in a radial fashion [see Fig. 4(b) for an example]. They possess long-range correlations and area-law entanglement, and photonic rp-PEPS can be efficiently prepared with the circuit depth, Eq. (1).

The generation procedure of rp-PEPS is shown in Fig. 5. We start from an initial state where all the cavities and the transmons are in their ground states $\left|\varphi_{0}\right\rangle=\left|0_{\left\{A_{j}\right\}}\right\rangle \otimes\left|0_{\left\{T_{j}\right\}}\right\rangle$, and sequentially apply unitaries $\left\{\hat{U}_{[i, j]}\right\}$. The unitary $U_{[i, j]}$ is applied in the $i$ th layer on the ancillas $\left\{A_{j}, \ldots, A_{j+L_{p}-1}\right\}$ and transmons $\left\{T_{j}, \ldots, T_{j+L_{p}-1}\right\}$. As each ancilla can be viewed effectively as $L_{p}-1$ qubits [Eq. (20)], the unitary equivalently acts on a plaquette of qubits of size $L_{p} \times L_{p}$. After each unitary, we trigger the photon emission from the transmon $T_{j}$ [denoted as isometry $M_{\mathrm{ph}}^{j}$; cf. Eq. (4)]. Note that after the last unitary per column, the last emission process per column $M_{\mathrm{ph}}^{m-L_{p}+1}$ (see step 4 of Fig. 5) converts excitations of transmons $\left\{T_{m-L_{p}+1}, \ldots, T_{m}\right\}$ to multiple photonic qubits at the same time. Repeatedly applying this procedure following the order shown in Fig. 5 [also see below in Eq. (21)], and in the end emitting the remaining excitations in the ancillas (an operation collectively denoted as $\mathcal{S}$ ), we generate the desired two-dimensional photonic state [36],

$$
\left|\psi_{\mathrm{rp}}\right\rangle=\left\langle\varphi_{0}\left|\mathcal{S} \prod_{i=1}^{n-L_{p}+1} \prod_{j=1}^{m-L_{p}+1}\left(M_{\mathrm{ph}}^{j} \hat{U}_{[i, j]}\right)\right| \varphi_{0}\right\rangle .
$$

Given the universal control of Eq. (19), this protocol can produce arbitrary states of the form of Eq. (21) [schematically shown in Fig. 4(b)], which are two-dimensional rp-PEPS of plaquette size $L_{p}$ with open boundary condition, with their source point located at the first photon being created [78]. Also note that, in the above protocol, photon emissions reset the transmon. This allows one to efficiently reuse them, and this parallelizes the preparation procedure (shown in steps 3 and 4 of Fig. 5), such that the circuit depth for preparing rp-PEPS of plaquette length $L_{p}$ on an $n \times m$ lattice asymptotically scales as Eq. (1) [36]. This system further allows increasing the plaquette size by increasing the number of cavity-transmon pairs, $L_{C}$, or using more modes $D^{\prime}$ in the cavity.

Finally, we note that by replacing the photon emissions with qubit measurements, this state generation protocol naturally becomes a qubit-efficient quantum variational scheme $[79,80]$.

\section{Examples}

\section{Two-dimensional cluster state}

Consider a two-dimensional square lattice, with the position vector of each site denoted as $\vec{a} \equiv(i, j)$. The twodimensional cluster state $|\mathrm{Cl}\rangle_{2 \mathrm{D}}$ is prepared starting from a product state of all qubits in $|+\rangle$ by applying controlled-Z (CZ)

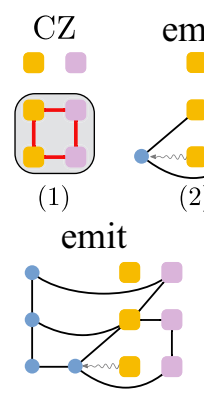

(6)

\section{emit}

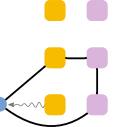

(2)

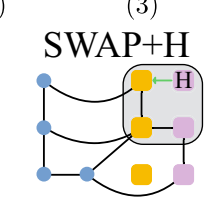

(7.1)

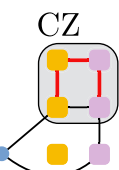

(3)

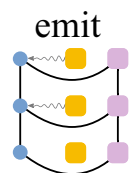

(4)

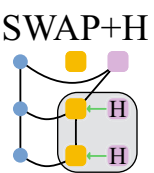

$(5.1)$ $\mathrm{CZ}$

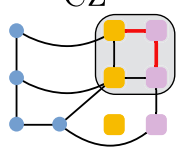

$(7.2)$
$\mathrm{CZ}$

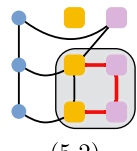

$(5.2)$
FIG. 6. Preparing two-dimensional cluster state $|\mathrm{Cl}\rangle_{2 \mathrm{D}}$ following the rp-PEPS preparation protocol (cf. Fig. 5, and we keep the same notation as there). Here both the ancillas and transmons have two levels (qubits), initialized in the state $|+\rangle$. The lines connecting two qubits mean that they have been acted on by a controlled-Z (CZ) gate in a previous step, whereas the thick red (gray) lines denotes that $\mathrm{CZ}$ gates are being applied in that step. Steps 1-4 prepare the first column of photons. To create the next column of photons, in each unitary we first swap certain ancilla states to the corresponding transmons in the same row [illustrated by green (gray) arrows], then apply Hadamard gates to set the ancilla states to $|+\rangle$ (see steps 5.1 and 7.1), and finally apply CZ gates, which prepares the second column (see step 8). By repeating steps 5.1 to 8 , one can prepare $|\mathrm{Cl}\rangle_{2 \mathrm{D}}$ of arbitrary size.

gates on each nearest-neighbor pairs of qubits [66]:

$$
|\mathrm{Cl}\rangle_{2 \mathrm{D}}=\prod_{\vec{b} \text { adjacent } \vec{a}} \mathrm{CZ}_{\vec{a} \vec{b}} \bigotimes_{\{\vec{a}\}}|+\rangle .
$$

This state is an rp-PEPS of plaquette size $L_{p}=2$; thus it can be prepared by the protocol in Sec. III B, where each ancilla consists of a qubit (this means that we use $L_{c}=1$ cavity with $D^{\prime}=2$ Fock basis). The preparation procedure is shown in Fig. 6, where the corresponding plaquette unitaries $\left\{U_{[i, j]}\right\}$ in Eq. (21) are formed by CZ, SWAP, and Hadamard gates.

One can easily extend the size of the state horizontally by repeatedly applying the steps 5.1-8 in Fig. 6, and vertically by fabricating a longer chain of ancilla-transmon pairs. Moreover, omitting some photon emissions, one can create arbitrary graph states of local connectivities in this way [23].

To prepare $|\mathrm{Cl}\rangle_{2 \mathrm{D}}$ of size $n \times m$, the depth for this circuit in terms of plaquette unitaries is $\mathcal{T} \approx 2 n+m$ [cf. Eq. (1)]. We also point out that this can be further improved to $\mathcal{T}_{\mathrm{cl}} \approx 2 n$, since the $\mathrm{CZ}$ gates commute with each other. As an example, steps $1-4$ in Fig. 6 can be combined such that we apply $\mathrm{CZ}$ gates on all adjacent pairs in the ancilla-transmon array in parallel (which are contained in two layers of plaquette unitaries), then emit one column of photons. The $\mathrm{CZ}$ gates between two cavities can be realized by combining singlequbit rotations and a controlled-NOT (CNOT) gate, which has been experimentally implemented in Ref. [81].

The above further parallelization of the circuit shows a generic feature of photonic rp-PEPS [Eq. (21)]: if the sequential product of unitaries $\hat{U}_{\text {col }}^{i}=\prod_{j=1}^{m-L_{p}+1} \hat{U}_{[i, j]}$ for preparing each column of photons can be parallelized as a circuit of depth $O(1)$, one can directly implement $\hat{U}_{\text {col }}^{i}$ followed by the photon emission of all transmon emitters $\otimes_{j} M_{\mathrm{ph}}^{j}$ to prepare 


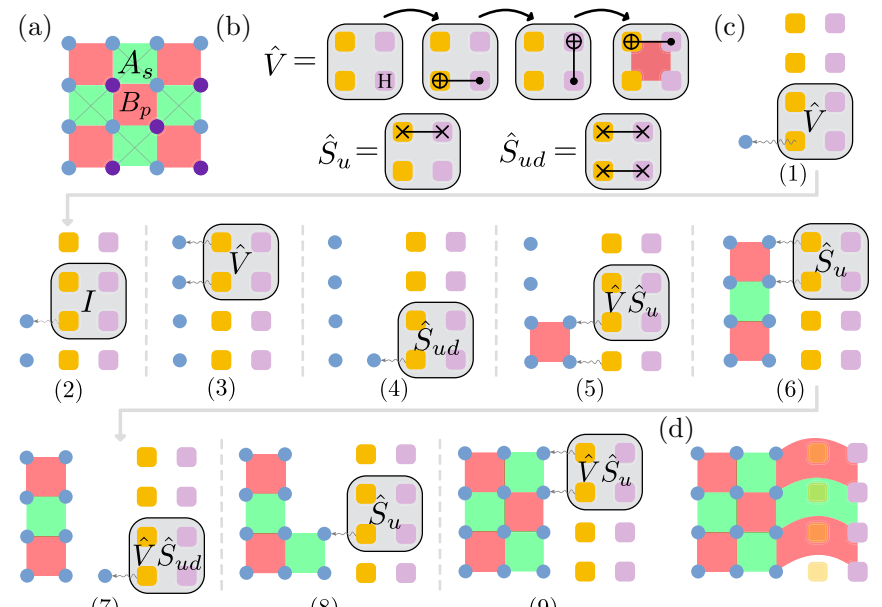

(7)

(8)

(9)

FIG. 7. Preparation of photonic toric code $|\mathrm{TC}\rangle$, inspired by Ref. [47]. Throughout this figure, we keep the same notation as that in Fig. 5. The support of the star operators $A_{s}$ is shown in green (light gray), and that of the plaquette operators $B_{p}$ in red (dark gray). (a) The toric code is the unique +1 eigenstate of all star and plaquette operators $A_{s}$ and $B_{p}$. Each plaquette has a "representative qubit," denoted by a purple dot. (b) We group the Hadamard and CNOT gates that will be applied to each plaquette as $\hat{V}$. The SWAP gates are denoted as $\hat{S}_{u}$ and $\hat{S}_{u d}$. (c) We alternatively apply $\hat{V}$ or identity gate $I$ in each step (dashed lines separate different steps), followed by a photon emission (for example, from steps 1 to 3). Also after each photon emission, one needs to swap the corresponding ancilla state to the transmon; thus in steps 4-9 the unitaries are generally the product of SWAP gates $\left(\hat{S}_{u}\right.$ or $\left.\hat{S}_{u d}\right)$ with $\hat{V}$ or $I$. (d) After step 9 of panel (c), one obtains $|\mathrm{TC}\rangle$ of size $4 \times 4$ with the same geometry as that in panel (a), consisting of three columns of photonic qubits and the column of ancilla qubits. By further repeating steps 4-9 in panel (c) and using more ancilla-transmon pairs, one can generate $|\mathrm{TC}\rangle$ of arbitrary size.

the $i$ th column of the rp-PEPS. This also applies to the toric code below.

\section{The toric code}

The toric code $[82,83]$ is an example of a string-net state, and finds important applications in quantum error correction. It is defined as the simultaneous +1 eigenstate of all star operators $A_{s}=\prod_{i \in s} Z_{i}$ [green boxes in Fig. 7(a)] and plaquette operators $B_{p}=\prod_{j \in p} X_{j}$ [red boxes in Fig. 7(a)], i.e., $A_{s}|\mathrm{TC}\rangle=B_{p}|\mathrm{TC}\rangle=+1|\mathrm{TC}\rangle$. Here $\left\{Z_{i}\right\}$ and $\left\{X_{j}\right\}$ are Pauli operators.

The toric code has recently been prepared on a stationary lattice, with the following procedure [47]:

(1) Initialize the whole lattice in the state $\bigotimes_{\{\vec{a}\}}|0\rangle$, where all $\left\langle A_{s}\right\rangle=1$ and $\left\langle B_{p}\right\rangle=0$.

(2) Choose a qubit for each plaquette as the representative qubit [an example choice is denoted by purple dots in Fig. 7(a)], and apply a Hadamard gate on it.

(3) Within each plaquette, sequentially apply CNOTs with the representative qubit as the control and other qubits as targets, with an ordering such that the representative qubits are not changed until the CNOTs in their plaquette have been applied [cf. Fig. 7(b)].
Inspired by this procedure, one can prepare $|\mathrm{TC}\rangle$ as a photonic rp-PEPS of $L_{p}=2$ using the protocol in Sec. III B, with each ancilla consisting of a single qubit. To ease the notation, in Fig. 7(b) we group the gates in steps 2 and 3 of the above procedures that act on each plaquette as $\hat{V}$, and the gates used to swap the ancilla and transmon state as $\hat{S}_{u}$ and $\hat{S}_{u d}$.

The preparation circuit is shown in Fig. 7(c), where the plaquette unitaries are alternatively formed by $\hat{V}$ or the identity gate $\hat{I}$, and their product with SWAP gates $\left(\hat{S}_{u}\right.$ or $\left.\hat{S}_{u d}\right)$. The $\hat{V}$ implement desired operations for each toric code plaquette region [47], while the photon emission and SWAP gates will "push" the produced entangled states toward the photon lattice (denoted by blue circles). After all operations in Fig. 7(c), one obtains $|\mathrm{TC}\rangle$ of size $4 \times 4$ on three columns of photonic qubits and the ancilla qubits, shown in Fig. 7(d). By further repeating steps 4-9 in Fig. 7(c) and using more ancilla-transmon pairs, one can generate $|\mathrm{TC}\rangle$ of arbitrary size. We also point out that, by further parallelizing the unitaries [47], one can apply the same procedure as described in the two-dimensional cluster state generation protocol (cf. Sec. III C 1) to obtain a circuit depth $\mathcal{T}_{\text {TC }} \approx 2 n$ in terms of plaquette unitaries for generating $|\mathrm{TC}\rangle$ of size $n \times m$.

The schemes presented here for cluster state generation and toric code generation are directly derived from their circuit generation on stationary lattices. This idea allows one to obtain photonic state generation circuits by utilizing existing circuits on stationary lattices. For example, one can generate string-net states by extending the protocol in Sec. III C 2, using similar circuits as in Ref. [84].

\section{Isometric tensor network states}

The rp-PEPS contain the isometric tensor network states [37] (isoTNS) as a subclass [36]. An isoTNS is parametrized by its bond dimension $D$ [37], which bounds the entanglement entropy of the state, and its physical dimension $d$, which specifies the Hilbert space dimension of each site.

As the protocol in Sec. III B can prepare rp-PEPS with the source point in the corner of the lattice, one can prepare the subclass of isoTNS whose orthogonality center (see details in Appendix E) is in this corner. To do so, one has to require the unitaries to have an "L" shape [36], as shown in Fig. 8 for the case of $D=2$ and $d=2$. Here each unitary $\hat{B}_{[i, j]}$ acts on the ancilla $A_{j}$ and transmons $\left\{T_{j}, \ldots, T_{j+L_{p}-1}\right\}$ to produce isoTNS of bond dimension $D \leqslant 2^{L_{p}-1}$. Increasing the isoTNS bond dimension corresponds to increasing the arm length of the L-shaped unitary. In the end of the protocol, we can disentangle the ancilla from the photonic state being produced [36]. We provide more details on isoTNS in Appendix E.

The subclass of isoTNS whose orthogonality center is in the corner of the lattice already contains all graph states of local connectivities [23] and all string-net states [43], and thus the two-dimensional cluster state and toric code discussed previously can be also created in this way. However, we point out that, the circuits we presented in Secs. III C 1 and III C 2 are more efficient than the circuit derived from their isoTNS representation. For example, the isoTNS representation of arbitrary $\mathbb{Z}_{\lambda}$ toric code [85] is shown in Appendix E, where the physical dimension of the tensor is $\lambda^{4}$. Thus for the qubit $(\lambda=2)$ toric code $(|\mathrm{TC}\rangle$ discussed in Sec. III C 2, this isoTNS 


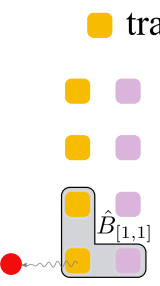

(1) transmon $\left\{T_{j}\right\}$

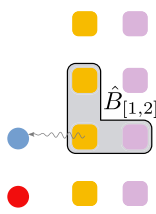

$(2)$

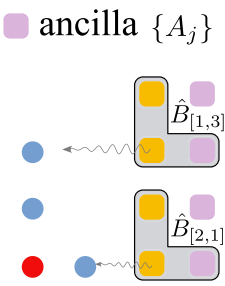

(3)

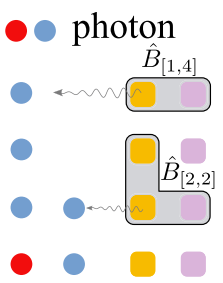

(4) repeated

FIG. 8. Preparation of isoTNS with bond dimension $D=2$ and physical dimension $d=2$. In the preparation of the $(i, j)$ th site, we apply a unitary $\hat{B}_{[i, j]}$ connecting the cavity $C_{j}$ and the transmons $T_{j}, T_{j+1}$, followed by a photon emission of the transmon $T_{j}$. After the initial steps ( 1 and 2), steps 3 and 4 will be repeated to build up the desired 2D isoTNS. The red (dark gray) dot denotes the orthogonality center of the isoTNS.

generation scheme requires each transmon emitter to be 16 dimensional, for which we need to couple multiple transmons to the same ancilla, and thus is more complex than the scheme shown in Sec. III C 2. The existence of the isoTNS representation of $\mathbb{Z}_{\lambda}$ toric code for arbitrary $\lambda$ also implies that our scheme can create photonic qudit toric code, and such states show certain advantages as quantum error-correcting codes compared to the qubit toric code [86,87].

Finally, the isoTNS is a class of states that can be efficiently prepared as shown here (also see Ref. [36]), and serve as an ansatz for classical variational algorithms [37]. Combining these two features may allow one to implement interesting protocols such as variational quantum metrology $[49,50,56]$.

\section{Scaling of rp-PEPS generation fidelity}

In the presence of imperfections, the rp-PEPS generation protocol (cf. Sec. III B) will yield a mixed photonic state $\rho_{\text {rp }}$. Here we provide a qualitative estimation of the fidelity $\mathcal{F}_{\mathrm{rp}}=$ $\left\langle\psi_{\mathrm{rp}}\left|\rho_{\mathrm{ph}}\right| \psi_{\mathrm{rp}}\right\rangle$ of the rp-PEPS generation protocol. Moreover, one can calculate the fidelity exactly by extending the MPDO approach in Appendix B.

Let us consider the array shown in Fig. 5(b) with $m$ rows, where each row consists of $L_{C}$ cavity-transmon pairs, with each cavity and transmon having the decoherence channels described in Sec. II C. Since we use the first $D^{\prime}$ Fock states of the cavity, we take the worst-case estimation of the cavity decay rate $\Gamma_{C}^{\prime}=\left(D^{\prime}-1\right) \Gamma_{C}$.

To prepare a generic rp-PEPS of size $n \times m$ and plaquette length $L_{p}\left(L_{p} \ll n, m\right)$, when preparing each photon we need to apply a unitary acting on $L_{p}^{2}$ qubits, thus $\operatorname{dim} \hat{U}_{[i, j]} \approx 2^{L_{p}^{2}}$. We estimate the time $T_{\mathrm{rp}}$ of implementing a generic unitary using optimal control methods as $T_{\mathrm{rp}} \sim\left(\operatorname{dim} \hat{U}_{[i, j]}\right)^{2}=O\left(4^{L_{p}^{2}}\right)$ [74]. Note that the numerical cost of the pulse optimization also increases exponentially with $L_{p}^{2}$. Since both the initial and the final states of the sources are the ground state $\left|\varphi_{0}\right\rangle$ [cf. Eq. (21)], in the preparation procedure (cf. Fig. 5), each source remains excited for a time $L_{p} n T_{\text {rp }}$. Moreover, there are in total around $\mathrm{nm}$ photon emissions. Thus by applying the same argument as in the MPS generation protocol (cf.
Sec. II C), one can estimate the scaling of the fidelity as

$$
\mathcal{F}_{\text {rp }} \sim \exp \left[-\xi_{\text {unit }}^{\prime D^{\prime}} \cdot m L_{c} \cdot L_{p} n T_{\text {rp }}-\left(\xi_{\mathrm{em}}^{\prime \mathrm{src}, D^{\prime}}+\xi_{\mathrm{em}}^{\mathrm{ph}}\right) \cdot n m\right],
$$

with $\xi_{\text {unit }}^{D^{\prime}}, \xi_{\mathrm{em}}^{\mathrm{src}, D^{\prime}}$, and $\xi_{\mathrm{em}}^{\mathrm{ph}}$ of the same form as that in Eqs. (13)-(15), but with cavity decay rate $\Gamma_{C}$ replaced by $\Gamma_{C}^{\prime}$ and different nonuniversal constants $\left\{\beta_{i}^{\prime}\right\}$.

Given $L_{p}$, the number of cavities in each ancilla $L_{C}$ is $L_{C}=\left\lceil\left(L_{p}-1\right) / \log _{2} D^{\prime}\right\rceil$. We can thus write overall scaling [Eq. (23)] as $\mathcal{F}_{\text {rp }} \sim \exp \left(-\xi_{\text {rp }}^{L_{p}, D^{\prime}} n m\right)$, with the error rate of generating each photon $\xi_{\mathrm{rp}}^{L_{p}, D^{\prime}}$ as

$$
\xi_{\mathrm{rp}}^{L_{p}, D^{\prime}} \approx 4^{L_{p}^{2}}\left(L_{p}-1\right) L_{p} \xi_{\mathrm{unit}}^{\prime D^{\prime}} / \log _{2} D^{\prime}+\xi_{\mathrm{em}}^{\prime \mathrm{src}, D^{\prime}}+\xi_{\mathrm{em}}^{\prime \mathrm{ph}} .
$$

From the above analysis, we see that using more cavities $L_{c}>1$ reduces the maximum number of excitations in each cavity $D^{\prime}=\left\lceil 2^{\left(L_{p}-1\right) / L_{c}}\right\rceil$ significantly, which leads to a corresponding reduction in the error due to cavity decay, $\Gamma_{C}^{\prime}=$ $\left(D^{\prime}-1\right) \Gamma_{C}$. In addition, it is experimentally easier to control cavities with small Hilbert space than controlling a single cavity with a large Hilbert space. However, the fidelity of the intercavity connection is generally lower than the singlecavity operation [77]. So depending on the desired plaquette length $L_{p}$ of the rp-PEPS, one needs to choose appropriate $L_{c}$ and $D^{\prime}$ to get the highest possible fidelity. A precise determination of the optimal $L_{c}$ and $D^{\prime}$ further depends on the specific target state, the details of the unitary operations, as well as the errors in the intercavity operations $[77,88]$.

We expect an experimental realization of the simplest $L_{c}=$ 1 protocol could be a good first step to further understand the performance of the rp-PEPS protocol. In the end, an experiment aiming at large plaquette sizes may have to be optimized regarding the total number of cavities used, but this is beyond the scope of the present paper.

\section{CONCLUSION}

We propose a physical platform and a protocol to sequentially generate microwave photonic tensor network states with moderately high bond dimensions based on a dispersively coupled cavity-transmon system. The good coherence properties of microwave cavities lead to favorable scaling of the photon number for the MPS; in particular, we show this platform can potentially create a one-dimensional cluster state of over a hundred photons deterministically with current technology. The good connectivity makes this platform a promising candidate for generating a large class of high-dimensional rp-PEPS, and we show how to create a two-dimensional cluster state, the toric code, and isoTNS as examples. Our work thus serves as systematic guidance for sequential photon generation experiments in circuit QED platforms, and can naturally be applied to other dispersively coupled qubit-oscillator systems.

Our work can be extended in many ways. First, there are plenty of ideas to further reduce the imperfections during the protocol by applying error-correction techniques [89,90], applying error-transparent gates or path-independent gates [91] on the bosonic modes, or applying open system optimal control techniques $[65,92,93]$. Second, the ability to generate strongly correlated photonic tensor network states opens the 
door of developing quantum information processing protocols that go beyond MPS $[49,50,56]$. One can further simultaneously use coupled arrays of emitters and non-Markovian feedback approaches [26-32] to reduce the component overhead of the system and possibly generate a larger class of photonic states.

\section{ACKNOWLEDGMENTS}

We thank Yujie Liu and Alejandro González-Tudela for their insightful discussions. We acknowledge funding from ERC Advanced Grant QUENOCOBA under the EU Horizon 2020 program (Grant Agreement No. 742102) and the European Union's Horizon 2020 research and innovation program under Grant No. 899354 (FET Open SuperQuLAN).

\section{APPENDIX A: MPS GENERATION WITH CIRCUIT QED USING QUANTUM OPTIMAL CONTROL APPROACH}

In this section, we introduce our quantum optimal control (QOC) approach (similar to that used in Ref. [13]) to implement unitaries on this setup.

We aim to find the driving amplitude $\varepsilon_{C}(t), \varepsilon_{T}(t)$ for the control $H_{\text {drive }}(t)$ [Eq. (3)] that implements the desired unitary operations $U_{[i]}$ [Eq. (5)] in $\mathcal{H}_{D} \otimes \mathcal{H}_{T}$.

Given the target MPS to be prepared [Eq. (6)], one can construct a series of isometries $\left\{\hat{A}_{[i]}\right\}$ that needs to be implemented on the system [9]. For generating the $n$-photon cluster state [Eq. (7)], this construction gives two kinds of isometries $\hat{A}_{[i \neq n]}$ and $\hat{A}_{[n]}$, as well as the ancilla initial state $\left|\varphi_{I}\right\rangle_{C}$ needed in the protocol [cf. Eq. (6)]:

$$
\begin{aligned}
\hat{A}_{[i \neq n]} & =\left(\begin{array}{c}
V_{[i]}^{0} \\
V_{[i]}^{1}
\end{array}\right)=\frac{1}{\sqrt{2}}\left(\begin{array}{rr}
1 & 0 \\
1 & 0 \\
0 & 1 \\
0 & -1
\end{array}\right), \\
\hat{A}_{[n]} & =\left(\begin{array}{ll}
1 & 0 \\
0 & 0 \\
0 & 1 \\
0 & 0
\end{array}\right), \\
\left|\varphi_{I}\right\rangle_{C} & =\frac{1}{\sqrt{2}}\left(|0\rangle_{C}+|1\rangle_{C}\right) .
\end{aligned}
$$

Note that here $\hat{A}_{[n]}$ is different from all other $\hat{A}_{[i \neq n]}$, as it contains an additional operation to disentangle the cavity from the photons.

We can embed above $\left\{\hat{A}_{[i]}\right\}$ into $U_{[i]}$ to realize Eq. (5) [see Eq. (A2), below]. In numerical calculations we keep the first $N_{C}>D$ Fock states in $\mathcal{H}_{C}$. Thus $\operatorname{dim} \mathcal{H}_{C}=N_{C}$ in our numerical calculation. One can then write $U_{[i]}$ as

$$
U_{[i]}=\left(\begin{array}{cc}
\hat{A}_{[i]} & B_{1} \\
O & B_{2}
\end{array}\right),
$$

with its basis vector permuted as

$$
\operatorname{Base}\left(U_{[i]}\right) \equiv\left[\operatorname{Base}\left(\mathcal{H}_{D} \otimes \mathcal{H}_{T}\right), \text { others }\right] .
$$

The $O$ is a zero matrix, which physically means that $U_{[i]}$ does not cause the population to leak out of $\mathcal{H}_{D} \otimes \mathcal{H}_{T}$. The parts $B_{1}$ and $B_{2}$ are arbitrary, as long as $U_{[i]}$ is a unitary of dimension $2 N_{C}$. One needs two kinds of unitaries. Each application of $U_{[i \neq n]}$ followed by the photon emission adds one site to the cluster state. The last unitary $U_{[n]}$ followed by a photon emission disentangles the source from the photonic MPS.

To apply QOC to our circuit QED (cQED) platform with Hamiltonian $H_{\text {src }}$ [Eqs. (2) and (3)], we go to the rotating frame to remove energy terms of the transmon and the cavity mode in $H_{\text {src }}$, getting

$$
H_{\mathrm{src}}^{\prime}(t)=\chi \sigma_{11} a^{\dagger} a+\left[\varepsilon_{C}(t) a+\varepsilon_{T}(t) \sigma_{01}+\text { H.c. }\right],
$$

and readily apply the QOC algorithm in Ref. [13] with the control Hamiltonian $H_{\mathrm{src}}^{\prime}(t)$ to find the pulse sequences to implement desired $U_{[i]}$. The pulse sequence to implement $U_{[i \neq n]}$ and $U_{[n]}$ for the cluster state generation are shown in Fig. 2, where we choose $N_{C}=5$.

\section{APPENDIX B: THE MATRIX PRODUCT DENSITY} OPERATOR APPROACH TO COMPUTE STATE FIDELITY

In this section we recall the MPDO approach [13] to compute the fidelity $\mathcal{F}_{\text {MPS }}$ of the photonic state. We can rewrite the master equation, Eq. (9), in a vectorized form:

$$
\frac{d \vec{\rho}_{\mathrm{src}}(t)}{d t}=\mathcal{L}(t) \vec{\rho}_{\mathrm{src}}(t), \quad \vec{\rho}_{\mathrm{src}}=\sum_{a, b=1}^{N_{h}} \rho_{a b}|a \otimes \bar{b}\rangle .
$$

Here $\mathcal{L}(t)$ is the Liouville operator, $|a\rangle$ is a basis element in $\mathcal{H}_{\text {src }}$, and $|\bar{a}\rangle$ represents its complex conjugate. The solution of Eq. (B1) is

$$
\vec{\rho}_{\mathrm{src}}(T)=\mathcal{T}\left\{e^{\int_{0}^{T} \mathcal{L}(t) d t}\right\} \vec{\rho}_{\mathrm{src}}(0)=W_{\mathcal{L}} \vec{\rho}_{\mathrm{src}}(0) .
$$

The photon emission process can be described by a process map

$$
W_{\mathrm{ph}}=\sum_{i, j=0}^{1} \sum_{a, b, c, d=1}^{N_{h}} W_{P, a b c d}^{i j}|c, \bar{d}, i, \bar{j}\rangle|a, \bar{b}\rangle,
$$

which maps $\vec{\rho}_{\text {src }}$ with vectorized basis $|a, \bar{b}\rangle$ to a systemphoton joint density matrix with vectorized basis $|c, \bar{d}, i, \bar{j}\rangle=$ $|c, \bar{d}\rangle \otimes|i, \bar{j}\rangle_{\mathrm{ph}}$. Thus each photon generation round results in a map from the joint density matrix $\vec{\rho}_{[k-1]}$ of $k-1$ photons and system to the joint density matrix $\vec{\rho}_{[k]}$ of $k$ photons and system:

$\vec{\rho}_{[k]}=\sum_{i_{k}, j_{k}=0}^{d-1} N_{[k]}^{i_{k}, j_{k}} \vec{\rho}_{[k-1]}, \quad$ with $\quad N_{[k]}^{i_{k}, j_{k}}=W_{\mathrm{ph}}^{i_{k} j_{k}} W_{\mathcal{L}_{[k]}}$.

The fidelity $\mathcal{F}_{\text {MPS }}$ can be efficiently evaluated as [13]

$$
\begin{aligned}
\mathcal{F}_{\text {MPS }}= & \sum_{\left\{i_{k}, j_{k}\right\}=0}^{1} \operatorname{Tr}\left[N_{[n]}^{i_{n}, j_{n}} \cdots N_{[1]}^{i_{1}, j_{1}} \tilde{B}\right] \\
& \times \operatorname{Tr}\left[\left(A_{[n]}^{j_{n}} \otimes \bar{A}_{[n]}^{i_{n}}\right) \cdots\left(A_{[1]}^{j_{1}} \otimes \bar{A}_{[1]}^{i_{1}}\right)(B \otimes B)\right],
\end{aligned}
$$

where we denote $\tilde{B} \equiv \sum_{\alpha=1}^{N_{h}}\left|\varphi_{I}^{\prime}\right\rangle\left\langle\alpha|\otimes| \varphi_{I}^{\prime}\right\rangle\left\langle\alpha|, B \equiv| \varphi_{I}\right\rangle\left\langle\varphi_{F}\right|$, and $\bar{A}$ as the complex conjugate of a matrix $A$. For higherdimensional rp-PEPS, the $\mathcal{F}_{\mathrm{rp}}$ can be computed in the same 
way by viewing the high-dimensional rp-PEPS as an MPS with bond dimension and physical dimension scaling exponentially with the number of sequential photon sources, $m$.

\section{APPENDIX C: CONSTRUCTION OF PROCESS MAP $W_{\mathrm{ph}}$}

The decoherence effects and the finite photon retrieval efficiency $p_{\mathrm{em}}$ during the photon emission will modify $W_{\mathrm{ph}}$ from its ideal form $W_{\mathrm{ph}}=M_{\mathrm{ph}} \otimes \bar{M}_{\mathrm{ph}}$ with $M_{\mathrm{ph}}$ of the form in Eq. (4). A good way to construct $W_{\text {ph }}$ is to include environmental photon modes which capture the erroneous jump events. When there is a finite photon retrieval efficiency $p_{\mathrm{em}}$, we can include an environmental photon mode $\varepsilon_{T}$, and the $M_{\mathrm{ph}}: \mathcal{H}_{T} \rightarrow \mathcal{H}_{T} \otimes \mathcal{H}_{\mathrm{ph}} \otimes \mathcal{H}_{\varepsilon_{T}}$ becomes

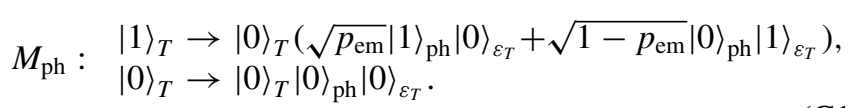

Here the label "ph" marks the desired photon mode, and $\varepsilon_{T}$ is an environmental mode that marks the erroneously emitted photon. We construct $W_{\mathrm{ph}}$ by $W_{\mathrm{ph}}=\operatorname{Tr}_{\varepsilon_{T}}\left[M_{\mathrm{ph}} \otimes \bar{M}_{\mathrm{ph}}\right]$, in which we trace out the $\varepsilon_{T}$ mode.

The effect of a transmon decay $\Gamma_{T}$ similarly leads to a branching of the emission, whereby $M_{\mathrm{ph}}: \mathcal{H}_{T} \rightarrow \mathcal{H}_{T} \otimes$ $\mathcal{H}_{\mathrm{ph}} \otimes \mathcal{H}_{\varepsilon_{T}}$ becomes

$$
\begin{aligned}
& M_{\mathrm{ph}}:|1\rangle_{T} \rightarrow|0\rangle_{T}\left(\sqrt{\frac{\Gamma_{\mathrm{em}}}{\Gamma_{\mathrm{em}}+\Gamma_{T}}}|1\rangle_{\mathrm{ph}}|0\rangle_{\varepsilon_{T}}+\sqrt{\frac{\Gamma_{T}}{\Gamma_{\mathrm{em}}+\Gamma_{T}}}|0\rangle_{\mathrm{ph}}|1\rangle_{\varepsilon_{T}}\right) \\
& |0\rangle_{T} \rightarrow|0\rangle_{T}|0\rangle_{\mathrm{ph}}|0\rangle_{\varepsilon_{T}} \text {. }
\end{aligned}
$$

The transmon dephasing leads to an exponential decay of the density matrix elements that are off diagonal on the transmon basis with rate $\Gamma_{\phi} / 2$. In the regime of $\Gamma_{\phi} \ll \Gamma_{\text {em }}$ the probability accumulates as

$$
\int_{0}^{T_{\mathrm{em}}} \frac{\Gamma_{\phi}}{2}\left\langle\sigma_{e g}(t)\right\rangle d t \approx \int_{0}^{\infty} \frac{\Gamma_{\phi}}{2} e^{-\Gamma_{\mathrm{em}} t / 2} d t=\Gamma_{\phi} / \Gamma_{\mathrm{em}}
$$

Thus, the mapping will lead to

$$
W_{\mathrm{ph}}^{10} \rightarrow\left(1-\frac{\Gamma_{\phi}}{\Gamma_{\mathrm{em}}}\right) W_{\mathrm{ph}}^{10}, \quad W_{\mathrm{ph}}^{01} \rightarrow\left(1-\frac{\Gamma_{\phi}}{\Gamma_{\mathrm{em}}}\right) W_{\mathrm{ph}}^{01}
$$

in Eq. (B3).

The cavity decay will explicitly depend on the photon emission time $T_{\mathrm{em}}$. Since $\Gamma_{C} \ll \Gamma_{\mathrm{em}}$, we can solve the dynamics analytically using the quantum trajectory approach [94] and include up to one jump process of the cavity photon. During the emission process, the decay probability for the Fock state $|n\rangle$ of the cavity mode is approximately $n \Gamma_{C} T_{\mathrm{em}}$. By including a cavity decay environmental mode $\varepsilon_{C}$, we can write $M_{\mathrm{ph}}: \mathcal{H}_{C} \otimes \mathcal{H}_{T} \rightarrow \mathcal{H}_{C} \otimes \mathcal{H}_{T} \otimes \mathcal{H}_{\mathrm{ph}} \otimes \mathcal{H}_{\varepsilon_{C}}$ as

$$
M_{\mathrm{ph}}: \begin{aligned}
& |n\rangle_{C}|1\rangle_{T} \rightarrow \sqrt{1-n \Gamma_{C} T_{\mathrm{em}}}|n\rangle_{C}|0\rangle_{T}|1\rangle_{\mathrm{ph}}|0\rangle_{\varepsilon_{C}}+\sqrt{n \Gamma_{C} T_{\mathrm{em}}}|n-1\rangle_{C}|0\rangle_{T}|1\rangle_{\mathrm{ph}}|1\rangle_{\varepsilon_{C}}, \\
& |n\rangle_{C}|0\rangle_{T} \rightarrow \sqrt{1-n \Gamma_{C} T_{\mathrm{em}}}|n\rangle_{C}|0\rangle_{T}|0\rangle_{\mathrm{ph}}|0\rangle_{\varepsilon_{C}}+\sqrt{n \Gamma_{C} T_{\mathrm{em}}}|n-1\rangle_{C}|0\rangle_{T}|0\rangle_{\mathrm{ph}}|1\rangle_{\varepsilon_{C}} .
\end{aligned}
$$

Similarly, we obtain $W_{\mathrm{ph}}$ by $W_{\mathrm{ph}}=\operatorname{Tr}_{\varepsilon_{C}}\left[M_{\mathrm{ph}} \otimes M_{\mathrm{ph}}^{\dagger}\right]$. A finite photon emission time also leads to a residue population on $p_{1 T}\left(T_{\mathrm{em}}\right)=e^{-\Gamma_{\mathrm{em}} T_{\mathrm{em}}} p_{1 T}(0)$ on the state $|1\rangle_{T}$. This can be modeled by a further reduction factor on $p_{\mathrm{em}}$ whereby $p_{\mathrm{em}} \rightarrow$ $\left(1-e^{-\Gamma_{\mathrm{em}} T_{\mathrm{em}}}\right) p_{\mathrm{em}}$.

With the above analysis, we can write down the $M_{\mathrm{ph}}: \mathcal{H}_{C} \otimes \mathcal{H}_{T} \rightarrow \mathcal{H}_{C} \otimes \mathcal{H}_{T} \otimes \mathcal{H}_{\mathrm{ph}} \otimes \mathcal{H}_{\varepsilon_{T}} \otimes \mathcal{H}_{\varepsilon_{C}}$ that includes all the above effects as

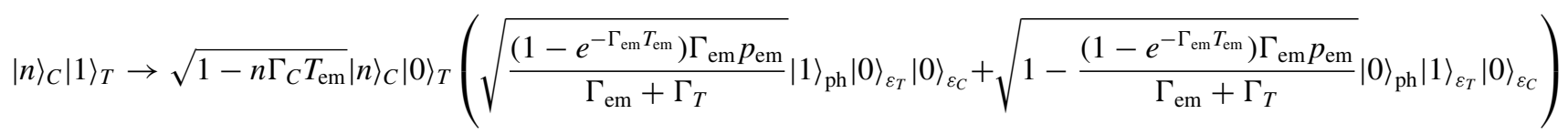

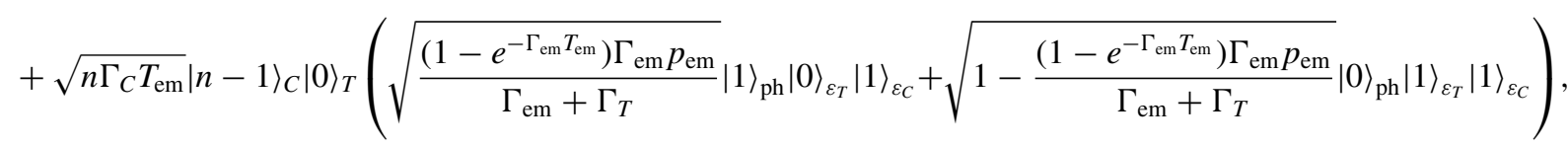

$$
\begin{aligned}
& |n\rangle_{C}|0\rangle_{T} \rightarrow \sqrt{1-n \Gamma_{C} T_{\mathrm{em}}}|n\rangle_{C}|0\rangle_{T}|0\rangle_{\mathrm{ph}}|0\rangle_{\varepsilon_{T}}|0\rangle_{\varepsilon_{C}}+\sqrt{n \Gamma_{C} T_{\mathrm{em}}}|n-1\rangle_{C}|0\rangle_{T}|0\rangle_{\mathrm{ph}}|0\rangle_{\varepsilon_{T}}|1\rangle_{\varepsilon_{C}} .
\end{aligned}
$$

And we further trace out the environmental modes to get $W_{\mathrm{ph}}=\operatorname{Tr}_{\varepsilon_{T}, \varepsilon_{C}}\left[M_{\mathrm{ph}} \otimes \bar{M}_{\mathrm{ph}}\right]$. After that, we include the transmon dephasing by applying Eq. (C4), to finish the construction of $W_{\mathrm{ph}}$.

\section{APPENDIX D: ADDITIONAL SCALING DATA FOR MPS PREPARATION FIDELITY}

\section{Scaling of the error rate $\chi$}

As shown in the main text, we can compute the scaling of the coefficient $\xi$ of the exponentially decaying fidelity
$\mathcal{F}_{\text {MPS }}=e^{-\xi n}$ with the slope (error rate) $\xi$ as a function of various imperfections. This scaling is numerically shown here in Figs. 9(a)-9(g), where each data point is extracted from a MPDO calculation of the relation between $\mathcal{F}_{\text {MPS }}$ and the photon number $n$ [an example is shown in Fig. 3(a)], with 

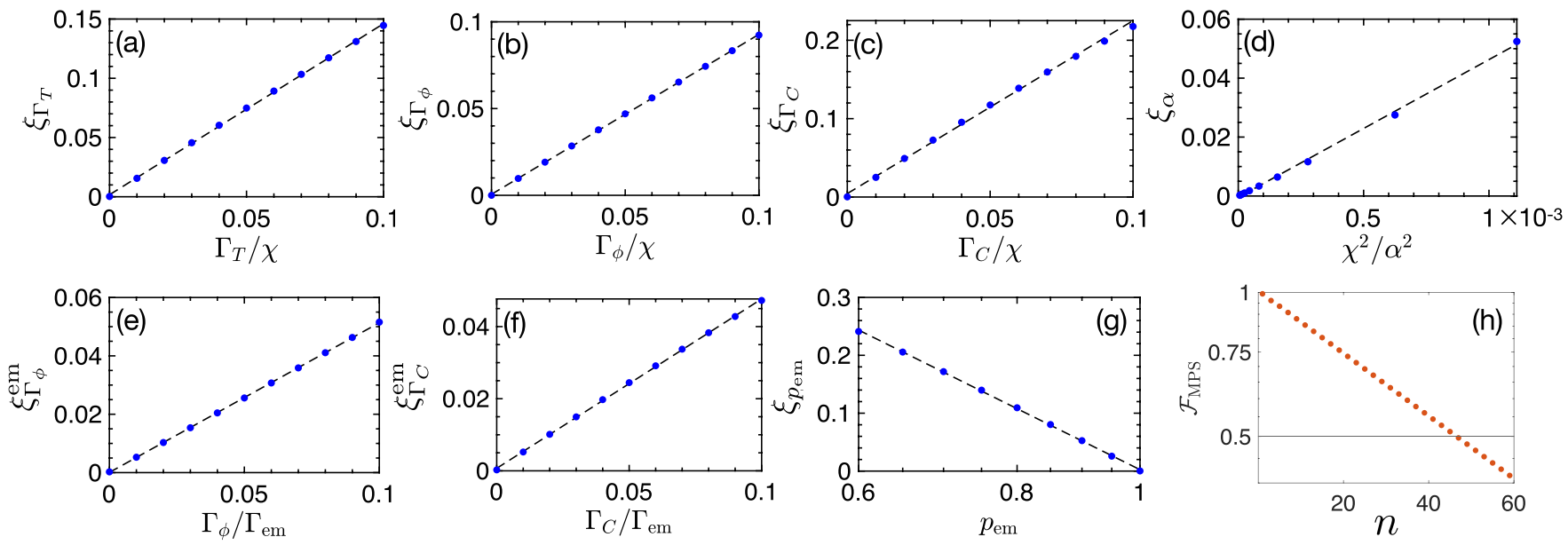

FIG. 9. Scaling of the error rate $\xi$ in $\mathcal{F}_{\text {MPS }}$ for creating the cluster state [Eq. (7)] using pulse sequence in Fig. 2 as a function of (a) the transmon decay during unitary operation, (b) the transmon dephasing during unitary operation, (c) the cavity decay during unitary operation, (d) the transmon anharmonicity during unitary operation, (e) the transmon dephasing during photon emission, (f) the cavity decay during photon emission, and (g) the photon retrieval efficiency. (h) The MPDO calculation of $\mathcal{F}_{\text {MPS }}$ versus the cluster state photon number $n$, with transmon parameters in section D2. The horizontal line denotes $\mathcal{F}_{\text {MPS }}=1 / 2$.

only the specific decoherence channel turned on. For example, in Fig. 9(a) only the transmon decay $\Gamma_{T}$ is turned on. From Figs. 9(a)-9(g) we obtain all individual terms in Eq. (12), with nonuniversal coefficients in Eq. (16) for the cluster state generation with the pulse sequence in Fig. 2. In the regime where the error per photon generation is small, we can estimate the total error $\xi$ by simply adding these individual terms, thus obtaining Eq. (12).

\section{Fidelity versus photon number for transmon parameters in Ref. [20]}

In Fig. 3(a) we showed the fidelity versus the cluster state photon number for the state-of-the-art experimental parameters shown in Sec. IID. To better compare it with the experimentally demonstrated result [20], here we compute the same relation with the transmon properties reported there, while other parameters stay the same as that in Sec. IID. Specifically, we choose $\Gamma_{T}=47.62 \mathrm{kHz}, \Gamma_{\phi}=58.82 \mathrm{kHz}$, $\alpha=2 \pi \times-303 \mathrm{MHz}$, and $\chi=2 \pi \times-5 \mathrm{MHz}$ [20]. The result is shown in Fig. 9(h), from which we get $N_{\text {ph }} \approx 47$.

\section{Achievable entanglement length $N_{\mathrm{ph}}$ with MPS bond dimension $D$}

The entanglement length $N_{\text {ph }}=\ln 2 / \xi$ is determined by the error rate per photon $\xi$ [Eq. (12)]. To produce an MPS with bond dimension $D$, we need to implement unitaries on $2 D$ dimensional Hilbert space. As numerical evidence suggesting that [74] the time cost of implementing a general unitary in $N$-dimensional Hilbert space using the quantum optimal control approach scales as $O\left(N^{2}\right)$, it takes $T_{\mathrm{MPS}}^{D}=O\left(D^{2}\right)$ to implement the above unitaries. This leads to increased decoherence as the coefficients $\beta_{C}, \beta_{T}, \beta_{\phi}$, and $\beta_{\alpha}$ in Eq. (8) are proportional to $T_{\mathrm{MPS}}^{D}$. In the regime of $p_{\mathrm{em}} \approx 1$ and $\beta_{0} \approx 0$ (typical for current experimental platforms [20,67]), one can thus estimate the scaling of $\xi$ as

$$
\xi \approx T_{\mathrm{MPS}}^{D} \xi_{\mathrm{unit}}+\xi_{\mathrm{em}}^{\mathrm{src}}+\xi_{\mathrm{em}}^{\mathrm{ph}} \sim O\left(D^{2}\right) .
$$

Thus the dominant part leads to a qualitative scaling of $N_{\mathrm{ph}} \sim D^{-2}$.

\section{APPENDIX E: ISOMETRIC TENSOR NETWORK STATES}

In this section, we provide more details on the definition of isometric tensor network states (isoTNS) [37]. To start, first we recall the definition of the projected entangled-pair states (PEPS) [33], which are defined through a network of tensors that are connected with each other, with one tensor at each lattice site [see Fig. 10(a)]. The wavefunction of PEPS is obtained by contracting the connected (virtual) legs of the tensors, as

$$
\left|\Psi_{\mathrm{PEPS}}\right\rangle=\sum_{\{k\}=0}^{d-1} \mathcal{F}_{2 \mathrm{D}}\left(\left\{B_{[i, j]_{\text {lurb }}}^{k}\right\}\right)|\{k\}\rangle,
$$

where the $B_{[i, j] l \text { lurd }}^{k}$ is a rank-5 tensor on the site $(i, j)$, which has virtual indices $l, u, r$, and $b$ of bond dimension $D$ and physical index $k$ of physical dimension $d$. And the symbol $\mathcal{F}_{2 D}$ denotes the contraction of the connected virtual indices. The PEPS serves as a natural extension of the MPS in higher dimensions, and has wide applications in describing higherdimensional many-body systems $[16,17,95]$.

The IsoTNS is a subclass of the PEPS, where the tensors satisfy certain isometry conditions. The isometry condition means that, when the incoming legs [denoted by the arrows in Fig. 10(b)] and the physical legs of a tensor are contracted with corresponding legs of the complex conjugate of this tensor, the remaining legs yield an identity. For example, the tensor in the dashed box in Fig. 10(b) obeys

$$
\sum_{k, u r} B_{[i, j]]_{l u r b}}^{k}\left(B_{[i, j]_{l^{\prime} u r b^{\prime}}^{k}}\right)^{*}=\delta_{b b^{\prime}} \delta_{l l^{\prime}},
$$

which is shown graphically in Fig. 10(c). Moreover, the two red shaded lines in Fig. 10(b) only have incoming arrows, which are termed the orthogonality hypersurface of the 
(a)

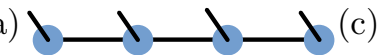
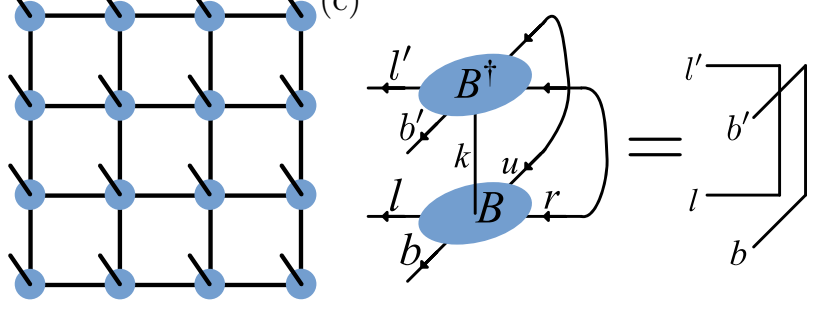

(b)
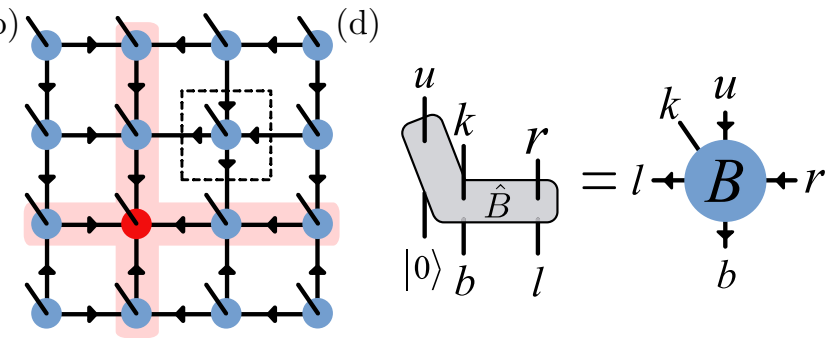

FIG. 10. The projected entangled pair states (PEPS) and isometric tensor network states (isoTNS). (a) PEPS are states represented by a network of tensors, where the tensor at each site is of physical dimension $d$, and is connected to that of the neighboring sites with virtual bonds of dimension $D$. (b) IsoTNS are a subclass of PEPS, where the tensors satisfy isometry conditions denoted by the arrows. The red (gray) shaded lines denote the orthogonality hypersurface of isoTNS, and their crossing point [the red (dark gray) dot] denotes the orthogonality center of isoTNS. (c) The isometry condition for the tensor inside the dashed box in panel (b). Here the incoming virtual legs and the physical legs of the tensor are contracted with the corresponding legs of the complex conjugate of the tensor, yielding identity on the outgoing legs. This is also mathematically shown in Eq. (E2). (d) One can map a three-qubit L-shaped quantum gate (see Fig. 8) to an isoTNS tensor of bond dimension $D=d$.

isoTNS, and their intersection is the orthogonality center of the isoTNS [37].

As shown in Ref. [36], one can convert an isoTNS tensor $B_{[i, j] l u r b}^{k}$ of the bond dimension $D$ into a ' $\mathrm{L}$ '-shaped unitary of the form

$$
\left.\hat{B}_{[i, j]}=\sum_{l u r b, k} B_{[i, j]}^{k}\right]_{l u r b}|u, k, r\rangle\langle 0, b, l|,
$$

where the indices are identified in Fig. 10(d) for the case of bond dimension $D=d$. Here the rank-5 tensor $B_{[i, j] \text { lurb }}^{k}$ also satisfies the same isometry condition, Eq. (E2). This shows that the gates in the photon generation scheme shown in Fig. 8 can be identified as isoTNS tensors. Increasing isoTNS bond dimension corresponds to increasing the arm length of the Lshaped unitary.

In this way, one can generate isoTNS by sequentially applying overlapping L-shaped unitaries, which lead to the photonic isoTNS generation protocol discussed in Sec. III C 3 . This fact further implies that isoTNS is a subclass of rp-PEPS [36] since we can cover the L-shapes unitaries by plaquette unitaries.

\section{IsoTNS representation of the toric code}

In general, the $\mathbb{Z}_{\lambda}$ toric code can be written as an isoTNS with the translational-invariant tensor at each site as [39]

$$
B_{l u r d}^{i_{1} i_{2} i_{3} i_{4}}=\frac{1}{\lambda} \delta_{l-u}^{i_{1}} \delta_{u-r}^{i_{2}} \delta_{r-b}^{i_{3}} \delta_{b-l}^{i_{4}},
$$

where

$$
\delta_{b}^{a}= \begin{cases}1 & \text { for } a=b \bmod \lambda \\ 0 & \text { otherwise. }\end{cases}
$$

This is a tensor of bond dimension $\lambda$ and physical dimension $\lambda^{4}$, and satisfies the isometry condition Eq. (E2).

\section{APPENDIX F: CONTROL UNIVERSALITY OF $\boldsymbol{H}_{\text {array }}(t)$}

Here we show the Hamiltonian $H_{\text {array }}(t)$ [Eq. (19)] can universally control the Hilbert space $\mathcal{H}_{\text {array }}^{L_{C} \times m}=\left(\mathcal{H}_{\text {src }}\right)^{\otimes L_{C} \times m}$. Let us consider case of two cQED sequential photon sources coupled to each other $\left(L_{C}=1\right.$ and $\left.m=2\right)$, whereby the whole Hilbert space is

$$
\mathcal{H}_{\text {array }}^{1 \times 2}=\mathcal{H}_{T} \otimes \mathcal{H}_{C} \otimes \mathcal{H}_{C} \otimes \mathcal{H}_{T}
$$

We know the Hamiltonian for each sequential photon source can control one $\mathcal{H}_{\text {src }}$ universally. This means one can create arbitrary Hamiltonians that act on each Hilbert space $\mathcal{H}_{C}$ of the cavity mode [see Eq. (F2), below]. Together with the bilinear interaction $H_{\text {int }}^{i j}$ [Eq. (18)] between two cavities, one can apply an analogous argument in Ref. [96], that by arithmetic operation and taking commutators between the bilinear coupling $H_{\text {int }}^{12}$ [cf. Eq. (18)] and single-cavity Hamiltonians

$$
H_{1}=\operatorname{poly}\left(a_{1}, a_{1}^{\dagger}\right), \quad H_{2}=\operatorname{poly}\left(a_{2}, a_{2}^{\dagger}\right),
$$

we can generate an arbitrary polynomial form of Hamiltonian,

$$
H_{12}=\operatorname{poly}\left(a_{1}, a_{1}^{\dagger}, a_{2}, a_{2}^{\dagger}\right) \in \mathcal{H}_{C} \otimes \mathcal{H}_{C} .
$$

This immediately implies that we can universally control the Hilbert space of two cavities [96]. Together with universal control on each sequential photon source, we can use Lemma 5.5 of Ref. [97] to combine the universality of two $\mathcal{H}_{\text {src }}$ and $\mathcal{H}_{C} \otimes \mathcal{H}_{C}$ to the whole $\mathcal{H}_{\text {array }}^{1 \times 2}$, which shows that we can universally control $\mathcal{H}_{\text {array }}^{1 \times 2}$ with $H_{\text {array }}(t)$ (here $L_{C}=1$ and $m=2$ ). By repeatedly applying Lemma 5.5 of Ref. [97], one can show $H_{\text {array }}(t)$ is further able to control $\mathcal{H}_{\text {array }}^{L_{C} \times m}$.
[1] J. L. O’Brien, A. Furusawa, and J. Vučković, Nat. Photonics 3, 687 (2009).

[2] N. Gisin and R. Thew, Nat. Photonics 1, 165 (2007).

[3] H. J. Kimble, Nature (London) 453, 1023 (2008).
[4] C. L. Degen, F. Reinhard, and P. Cappellaro, Rev. Mod. Phys. 89, 035002 (2017).

[5] D. C. Burnham and D. L. Weinberg, Phys. Rev. Lett. 25, 84 (1970). 
[6] H. S. Zhong, Y. Li, W. Li, L. C. Peng, Z. E. Su, Y. Hu, Y. M. He, X. Ding, W. Zhang, H. Li, L. Zhang, Z. Wang, L. You, X. L. Wang, X. Jiang, L. Li, Y. A. Chen, N. L. Liu, C. Y. Lu, and J. W. Pan, Phys. Rev. Lett. 121, 250505 (2018).

[7] K. M. Gheri, C. Saavedra, P. Törmä, J. I. Cirac, and P. Zoller, Phys. Rev. A 58, R2627 (1998).

[8] C. Saavedra, K. M. Gheri, P. Törmä, J. I. Cirac, and P. Zoller, Phys. Rev. A 61, 062311 (2000).

[9] C. Schön, E. Solano, F. Verstraete, J. I. Cirac, and M. M. Wolf, Phys. Rev. Lett. 95, 110503 (2005).

[10] C. Schön, K. Hammerer, M. M. Wolf, J. I. Cirac, and E. Solano, Phys. Rev. A 75, 032311 (2007).

[11] N. H. Lindner and T. Rudolph, Phys. Rev. Lett. 103, 113602 (2009).

[12] K. Tiurev, M. H. Appel, P. L. Mirambell, M. B. Lauritzen, A. Tiranov, P. Lodahl, and A. S. Sørensen, arXiv:2007.09295.

[13] Z.-Y. Wei, D. Malz, A. González-Tudela, and J. I. Cirac, Phys. Rev. Research 3, 023021 (2021).

[14] D. Perez-Garcia, F. Verstraete, M. M. Wolf, and J. I. Cirac, Quantum Inf. Comput. 7, 401 (2007).

[15] U. Schollwöck, Ann. Phys. 326, 96 (2011).

[16] R. Orús, Ann. Phys. 349, 117 (2014).

[17] J. I. Cirac, D. Perez-Garcia, N. Schuch, and F. Verstraete, Rev. Mod. Phys. 93, 045003 (2021).

[18] I. Schwartz, D. Cogan, E. R. Schmidgall, Y. Don, L. Gantz, O. Kenneth, N. H. Lindner, and D. Gershoni, Science 354, 434 (2016).

[19] C. Eichler, J. Mlynek, J. Butscher, P. Kurpiers, K. Hammerer, T. J. Osborne, and A. Wallraff, Phys. Rev. X 5, 041044 (2015).

[20] J.-C. Besse, K. Reuer, M. C. Collodo, A. Wulff, L. Wernli, A. Copetudo, D. Malz, P. Magnard, A. Akin, M. Gabureac, G. J. Norris, J. I. Cirac, A. Wallraff, and C. Eichler, Nat. Commun. 11, 4877 (2020).

[21] S. E. Economou, N. Lindner, and T. Rudolph, Phys. Rev. Lett. 105, 093601 (2010).

[22] M. Gimeno-Segovia, T. Rudolph, and S. E. Economou, Phys. Rev. Lett. 123, 070501 (2019).

[23] A. Russo, E. Barnes, and S. E. Economou, New J. Phys. 21, 055002 (2019).

[24] R. Bekenstein, I. Pikovski, H. Pichler, E. Shahmoon, S. F. Yelin, and M. D. Lukin, Nat. Phys. 16, 676 (2020).

[25] S. Bartolucci, P. Birchall, H. Bombin, H. Cable, C. Dawson, M. Gimeno-Segovia, E. Johnston, K. Kieling, N. Nickerson, M. Pant, F. Pastawski, T. Rudolph, and C. Sparrow, arXiv:2101.09310.

[26] H. Pichler, S. Choi, P. Zoller, and M. D. Lukin, Proc. Natl. Acad. Sci. USA 114, 11362 (2017).

[27] I. Dhand, M. Engelkemeier, L. Sansoni, S. Barkhofen, C. Silberhorn, and M. B. Plenio, Phys. Rev. Lett. 120, 130501 (2018).

[28] S. Xu and S. Fan, APL Photonics 3, 116102 (2018).

[29] K. Wan, S. Choi, I. H. Kim, N. Shutty, and P. Hayden, PRX Quantum 2, 040345 (2021).

[30] Y. Zhan and S. Sun, Phys. Rev. Lett. 125, 223601 (2020).

[31] Y. Shi and E. Waks, Phys. Rev. A 104, 013703 (2021).

[32] H. Bombin, I. H. Kim, D. Litinski, N. Nickerson, M. Pant, F. Pastawski, S. Roberts, and T. Rudolph, arXiv:2103.08612.

[33] F. Verstraete and J. I. Cirac, arXiv:cond-mat/0407066.

[34] F. Verstraete, M. M. Wolf, D. Perez-Garcia, and J. I. Cirac, Phys. Rev. Lett. 96, 220601 (2006).
[35] N. Schuch, M. M. Wolf, F. Verstraete, and J. I. Cirac, Phys. Rev. Lett. 98, 140506 (2007).

[36] Z.-Y. Wei, D. Malz, and J. I. Cirac, Phys. Rev. Lett. 128, 010607 (2022).

[37] M. P. Zaletel and F. Pollmann, Phys. Rev. Lett. 124, 037201 (2020).

[38] M. Hein, J. Eisert, and H. J. Briegel, Phys. Rev. A 69, 062311 (2004)

[39] N. Schuch, I. Cirac, and D. Pérez-García, Ann. Phys. 325, 2153 (2010).

[40] M. A. Levin and X. G. Wen, Phys. Rev. B 71, 045110 (2005).

[41] Z.-C. Gu, M. Levin, B. Swingle, and X.-G. Wen, Phys. Rev. B 79, 085118 (2009).

[42] O. Buerschaper, M. Aguado, and G. Vidal, Phys. Rev. B 79, 085119 (2009).

[43] T. Soejima, K. Siva, N. Bultinck, S. Chatterjee, F. Pollmann, and M. P. Zaletel, Phys. Rev. B 101, 085117 (2020)

[44] Note that the string-net states are originally defined on the hexagonal lattice [40]. Since one can embed a hexagonal lattice into a square lattice, one can create string-net states using the schemes we show in this paper, which are based on square lattices.

[45] Y. Takeuchi, T. Morimae, and M. Hayashi, Sci. Rep. 9, 13585 (2019).

[46] W. Asavanant, Y. Shiozawa, S. Yokoyama, B. Charoensombutamon, H. Emura, R. N. Alexander, S. Takeda, J. I. Yoshikawa, N. C. Menicucci, H. Yonezawa, and A. Furusawa, Science 366, 373 (2019).

[47] K. J. Satzinger, Y.-J Liu, A. Smith, C. Knapp, M. Newman, C. Jones, Z. Chen, C. Quintana, X. Mi, A. Dunsworth, C. Gidney, I. Aleiner, F. Arute, K. Arya, J. Atalaya, R. Babbush, J. C. Bardin, R. Barends, J. Basso, A. Bengtsson et al., Science 374, 1237 (2021).

[48] G. Semeghini, H. Levine, A. Keesling, S. Ebadi, T. T. Wang, D. Bluvstein, R. Verresen, H. Pichler, M. Kalinowski, R. Samajdar, A. Omran, S. Sachdev, A. Vishwanath, M. Greiner, V. Vuletic, and M. D. Lukin, Science 374, 1242 (2021).

[49] M. Jarzyna and R. Demkowicz-Dobrzański, Phys. Rev. Lett. 110, 240405 (2013).

[50] K. Chabuda, J. Dziarmaga, T. J. Osborne, and R. DemkowiczDobrzański, Nat. Commun. 11, 250 (2020).

[51] J. Miguel-Ramiro, A. Pirker, and W. Dür, npj Quantum Inf. 7, 135 (2021).

[52] Y. Huang, arXiv:1505.00772.

[53] A. M. Dalzell and F. G. Brandão, Quantum 3, 187 (2019).

[54] Y. Huang, arXiv:1903.10048.

[55] N. Schuch and F. Verstraete, arXiv:1711.06559.

[56] B. Koczor, S. Endo, T. Jones, Y. Matsuzaki, and S. C. Benjamin, New J. Phys. 22, 083038 (2020).

[57] K. Azuma, K. Tamaki, and H. K. Lo, Nat. Commun. 6, 6787 (2015).

[58] C. J. Axline, L. D. Burkhart, W. Pfaff, M. Zhang, K. Chou, P. Campagne-Ibarcq, P. Reinhold, L. Frunzio, S. M. Girvin, L. Jiang, M. H. Devoret, and R. J. Schoelkopf, Nat. Phys. 14, 705 (2018)

[59] P. Kurpiers, P. Magnard, T. Walter, B. Royer, M. Pechal, J. Heinsoo, Y. Salathé, A. Akin, S. Storz, J. C. Besse, S. Gasparinetti, A. Blais, and A. Wallraff, Nature (London) 558, 264 (2018). 
[60] P. Magnard, S. Storz, P. Kurpiers, J. Schar, F. Marxer, J. Lutolf, T. Walter, J.C. Besse, M. Gabureac, K. Reuer, A. Akin, B. Royer, A. Blais, and A. Wallraff, Phys. Rev. Lett. 125, 260502 (2020).

[61] M. Reagor, W. Pfaff, C. Axline, R. W. Heeres, N. Ofek, K. Sliwa, E. Holland, C. Wang, J. Blumoff, K. Chou, M. J. Hatridge, L. Frunzio, M. H. Devoret, L. Jiang, and R. J. Schoelkopf, Phys. Rev. B 94, 014506 (2016).

[62] Here we neglected the higher-order Hamiltonian terms such as the Kerr nonlinearity [67]. In principle, one can also include these terms in the pulse optimization.

[63] F. W. Strauch, Phys. Rev. Lett. 109, 210501 (2012).

[64] S. Krastanov, V. V. Albert, C. Shen, C. L. Zou, R. W. Heeres, B. Vlastakis, R. J. Schoelkopf, and L. Jiang, Phys. Rev. A 92 , 040303(R) (2015).

[65] S. Machnes, E. Assémat, D. Tannor, and F. K. Wilhelm, Phys. Rev. Lett. 120, 150401 (2018).

[66] H. J. Briegel and R. Raussendorf, Phys. Rev. Lett. 86, 910 (2001).

[67] R. W. Heeres, P. Reinhold, N. Ofek, L. Frunzio, L. Jiang, M. H. Devoret, and R. J. Schoelkopf, Nat. Commun. 8, 94 (2017).

[68] Note that the thermal excitations (photon gain) in the cavity and the transmon are neglected here.

[69] K. S. Chou, J. Z. Blumoff, C. S. Wang, P. C. Reinhold, C. J. Axline, Y. Y. Gao, L. Frunzio, M. H. Devoret, L. Jiang, and R. J. Schoelkopf, Nature (London) 561, 368 (2018).

[70] The photon retrieval efficiency $p_{\text {em }}$ depends on the explicit realization of the on-demand photon emission process, which we do not specify in this work. For example, in Ref. [20] the system is modeled with $p_{\mathrm{em}}=1$.

[71] P. Rebentrost and F. K. Wilhelm, Phys. Rev. B 79, 060507 (2009).

[72] Here we assume that the realization of our protocol using a cavity + transmon system can also be modeled with the photon retrieval efficiency $p_{\mathrm{em}}=1$, the same as that in Ref. [20].

[73] Since the emitter is always in its ground state at the start of each unitary operation (i.e., after each photon emission), the transmon will only be transiently populated during each unitary operation.

[74] J. Lee, C. Arenz, H. Rabitz, and B. Russell, New J. Phys. 20, 063002 (2018).

[75] Here we do not include the transmons in the ancilla space due to their relatively short coherence time compared to the photons in the cavities. However, in principle, one can include the transmons in the ancilla as well to further increase its Hilbert space dimension.
[76] C. Wang, Y. Y. Gao, P. Reinhold, R. W. Heeres, N. Ofek, K. Chou, C. Axline, M. Reagor, J. Blumoff, K. M. Sliwa, L. Frunzio, S. M. Girvin, L. Jiang, M. Mirrahimi, M. H. Devoret, and R. J. Schoelkopf, Science 352, 1087 (2016).

[77] Y. Y. Gao, B. J. Lester, Y. Zhang, C. Wang, S. Rosenblum, L. Frunzio, L. Jiang, S. M. Girvin, and R. J. Schoelkopf, Phys. Rev. X 8, 021073 (2018).

[78] The source point of the rp-PEPS is precisely defined as the location of the first plaquette unitary [36].

[79] W. Huggins, P. Patil, B. Mitchell, K. Birgitta Whaley, and E. Miles Stoudenmire, Quantum Sci. Technol. 4, 024001 (2019).

[80] J.-G. Liu, Y.-H. Zhang, Y. Wan, and L. Wang, Phys. Rev. Research 1, 023025 (2019).

[81] S. Rosenblum, Y. Y. Gao, P. Reinhold, C. Wang, C. J. Axline, L. Frunzio, S. M. Girvin, L. Jiang, M. Mirrahimi, M. H. Devoret, and R. J. Schoelkopf, Nat. Commun. 9, 652 (2018).

[82] E. Dennis, A. Kitaev, A. Landahl, and J. Preskill, J. Math. Phys. 43, 4452 (2002).

[83] A. Y. Kitaev, Ann. Phys. 303, 2 (2003).

[84] Y.-J. Liu, K. Shtengel, A. Smith, and F. Pollmann, arXiv:2110.02020.

[85] S. S. Bullock and G. K. Brennen, J. Phys. A: Math. Theor. 40, 3481 (2007).

[86] G. Duclos-Cianci and D. Poulin, Phys. Rev. A 87, 062338 (2013).

[87] F. H. E. Watson, H. Anwar, and D. E. Browne, Phys. Rev. A 92 , 032309 (2015).

[88] Y. Zhang, B. J. Lester, Y. Y. Gao, L. Jiang, R. J. Schoelkopf, and S. M. Girvin, Phys. Rev. A 99, 012314 (2019).

[89] N. Ofek, A. Petrenko, R. Heeres, P. Reinhold, Z. Leghtas, B. Vlastakis, Y. Liu, L. Frunzio, S. M. Girvin, L. Jiang, M. Mirrahimi, M. H. Devoret, and R. J. Schoelkopf, Nature (London) 536, 441 (2016).

[90] P. Campagne-Ibarcq, A. Eickbusch, S. Touzard, E. ZalysGeller, N. E. Frattini, V. V. Sivak, P. Reinhold, S. Puri, S. Shankar, R. J. Schoelkopf, L. Frunzio, M. Mirrahimi, and M. H. Devoret, Nature (London) 584, 368 (2020).

[91] W.-L. Ma, M. Zhang, Y. Wong, K. Noh, S. Rosenblum, P. Reinhold, R. J. Schoelkopf, and L. Jiang, Phys. Rev. Lett. 125, 110503 (2020).

[92] T. Schulte-Herbrüggen, A. Spörl, N. Khaneja, and S. J. Glaser, J. Phys. B: At. Mol. Opt. Phys. 44, 154013 (2011).

[93] M. Abdelhafez, D. I. Schuster, and J. Koch, Phys. Rev. A 99, 052327 (2019).

[94] M. B. Plenio and P. L. Knight, Rev. Mod. Phys. 70, 101 (1998).

[95] R. Orús, Nat. Rev. Phys. 1, 538 (2019).

[96] S. Lloyd and S. L. Braunstein, Phys. Rev. Lett. 82, 1784 (1999).

[97] T. Hofmann and M. Keyl, arXiv:1712.07613. 